This article was downloaded by: [The Royal Library]

On: 25 May 2010

Access details: Access Details: [subscription number 912937964]

Publisher Taylor \& Francis

Informa Ltd Registered in England and Wales Registered Number: 1072954 Registered office: Mortimer House, 3741 Mortimer Street, London W1T 3JH, UK

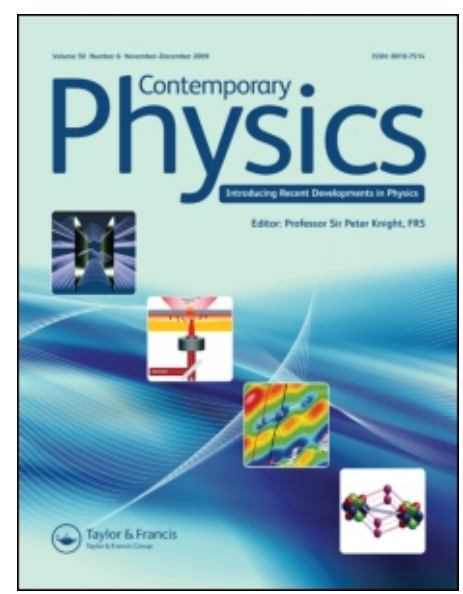

\title{
Contemporary Physics
}

Publication details, including instructions for authors and subscription information:

http://www.informaworld.com/smpp/title $\sim$ content=t713394025

\section{Climate transitions on long timescales}

Peter D. Ditlevsen ${ }^{a}$

${ }^{a}$ Centre for Ice and Climate, The Niels Bohr Institute, University of Copenhagen, Copenhagen,

Denmark

To cite this Article Ditlevsen, Peter D.(2009) 'Climate transitions on long timescales', Contemporary Physics, 50: 4, 511 532

To link to this Article: DOI: $10.1080 / 00107510902840313$

URL: http://dx.doi.org/10.1080/00107510902840313

\section{PLEASE SCROLL DOWN FOR ARTICLE}

Full terms and conditions of use: http://www.informaworld.com/terms-and-conditions-of-access.pdf

This article may be used for research, teaching and private study purposes. Any substantial or systematic reproduction, re-distribution, re-selling, loan or sub-licensing, systematic supply or distribution in any form to anyone is expressly forbidden.

The publisher does not give any warranty express or implied or make any representation that the contents will be complete or accurate or up to date. The accuracy of any instructions, formulae and drug doses should be independently verified with primary sources. The publisher shall not be liable for any loss, actions, claims, proceedings, demand or costs or damages whatsoever or howsoever caused arising directly or indirectly in connection with or arising out of the use of this material. 


\title{
Climate transitions on long timescales
}

\author{
Peter D. Ditlevsen* \\ Centre for Ice and Climate, The Niels Bohr Institute, University of Copenhagen, Juliane Maries Vej 30, DK-2100 \\ Copenhagen, Denmark
}

(Received 30 January 2009; final version received 20 February 2009)

\begin{abstract}
The climate has changed through the history of the Earth as evidenced in the geological records. Today we might be experiencing a climate change of the same magnitude as the transition into an ice age caused by very rapid burning and emission to the atmosphere of a substantial part of the fossilised carbon. Whether this leads to a gradual warming or if we will experience a transition into a different climatic state is presently unknown. The present day state-of-the-art numerical climate models are capable of producing fair representations of the current climate and are as such trusted to also predict the climate changes due to increasing atmospheric concentrations of greenhouse gases. However, the models are not presently capable of reproducing the rapid transitions from one climatic state, such as a glacial climate, into another, such as the present climate. The reason for this is unknown. The transitions are inherently 'non-linear' and thus not accessible through linear response theory. The term 'non-linear' is in this context defined as the phenomenon that the response of the system to a change in the forcing of the system is not linearly proportional to the forcing. This would happen if a threshold is reached such that the state of the system becomes unstable and the system bifurcates into a different state. There are strong indications in the geological records of this kind of behaviour for the climate. These dynamics can be understood in the context of fairly simple models of the climate.
\end{abstract}

Keywords: climate change; climate models

\section{Introduction}

The climate is governed by a hierarchy of physical processes determining how the incoming solar radiation and the outgoing long wave radiation are balanced. A very useful way of viewing the dynamics of the climate system is through analysing the energy flows. The incoming solar radiation is partly scattered back into space, partly absorbed into the atmosphere and partly absorbed into the surface of the planet. The surface is thus heated and subsequently releasing heat into the atmosphere both directly (sensible heat) and through evaporation of water, which release the heat while re-condensing in clouds (latent heat). The incoming energy flux to the surface depends on latitude through the variation in inclination of the Sun, thus 'inclination' and 'climate' have the same ontological roots. The differential heating leads to the generation of mechanical energy through the buoyancy forces caused by the expansion of air due to heating. This in turn redistributes the heat vertically through convection and horizontally through the winds. The mechanical energy is dissipated mainly through surface drags, some deposited as ocean surface waves, while at the end the energy is lost to space through long wave black-body radiation. The connection between the long wave radiation and the surface temperature depends on the vertical structure of the atmosphere, cloud processes and the amount of greenhouse gases in the atmosphere.

We shall in a loose sense consider the climate state to be the statistically steady state represented by some well defined variable, such as the global mean surface temperature. Already in doing this we must be cautious, since in principle, the system could reside in distinct climatic states, i.e. characterised by different meridional (equator to pole) temperature gradients with the same mean temperature. With this pragmatic definition of the climate the very first question one would ask is: 'What determines the mean surface temperature?'. Secondly, if this is indeed determined by, say, the incoming solar radiation: "What makes it stable and what makes it change?'. These questions turns out to be much harder to answer than what one would perhaps expect. A comparison of the presentday surface temperatures on Venus, Earth and Mars shows that these differ much more than what can be explained by the distances from the planets to the Sun. Figure 1 shows the solar flux as a function of the distance to the Sun. The curve shows what the surface temperature would be if conditions were as they are on

\footnotetext{
*Email: pditlev@gfy.ku.dk 


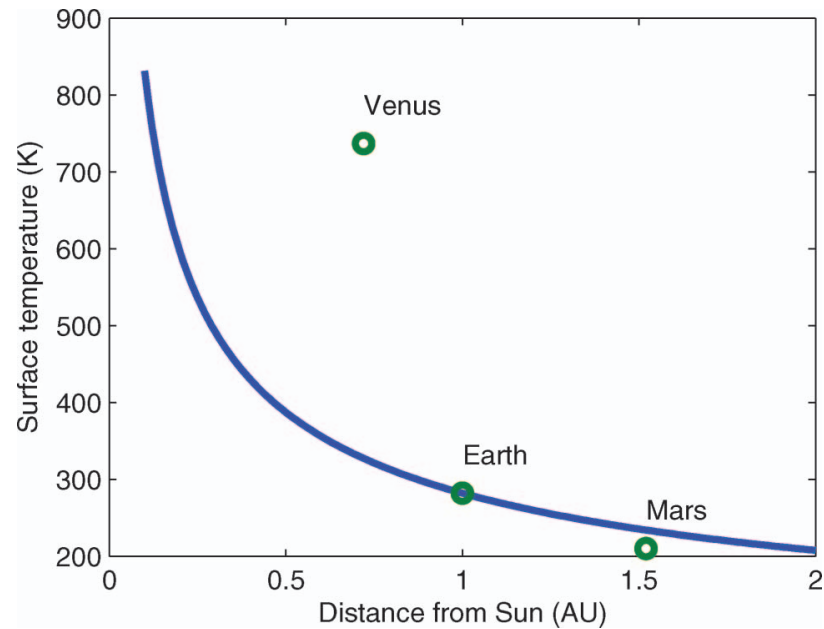

Figure 1. The surface temperature on a planet as a function of distance to the Sun. The curve shows how the temperature would be if conditions were identical to those of the Earth. Venus is much warmer due to an extremely thick greenhouse atmosphere. Mars is colder even though its atmosphere contains mainly $\mathrm{CO}_{2}$. That is because the atmosphere is very thin, only about $1 \%$ of that on Earth.

the Earth for planets at a given distance. As shown, the surface temperature on Venus is about $400 \mathrm{~K}$ warmer and Mars about $20 \mathrm{~K}$ colder than should be expected. This is an illustration that the temperature strongly depends on the planetary atmosphere and is not set solely by the incoming solar flux. The incoming solar flux has, due to the increase with time of nuclear fission in the interior of the star, risen by about a third during the lifetime of the solar system. This has remarkably not caused a drastic change in the surface temperature on Earth. On the contrary, there is good evidence for the existence of liquid water on the surface of the Earth for more than 4 billion years. This indicates that there are some mechanisms in the system to stabilise the existing climate state over long geologic times. Today we have pretty good ideas of what these mechanisms are.

Moving from the billion years timescale to the tens of millions of years, there is disturbing evidence of global glaciations, with freezing all the way to the equator, happening a few times and lasting for tens of millions of years just prior to the development of multicellular life. These are the so-called Snowball Earth events, which we shall see to be a consequence of multiple steady states in the radiative balance of the Earth. Since the last Snowball Earth event the climate has been warm and stable enough for the development of complex life forms.

For the last 20 million years the Earth has been so cold, that permanent ice sheets are found at the poles. Antarctica especially has been permanently ice covered. This is due to an unprecedented low atmospheric concentration of the greenhouse gas $\mathrm{CO}_{2}$. This climatic period is sometimes dubbed the 'Icehouse World' (in contrast to the 'Greenhouse World'). The Icehouse World is characterised by periodic waxing and waning of the large glaciers between ice ages and warm periods. It has long been known that the coming and going of ice ages is related to periodic changes due to the perturbations from the other planets in Earth's Keplerian orbit around the Sun. However, the climatic response to the changing solar insolation is not completely understood. As we shall see later this is also a strongly non-linear response phenomenon.

Finally, ice core records show that even within the last glacial period there are variations at the thousands of year timescale with abrupt jumps between two distinct quasi-stable climate states. These jumps apparently occur at random which indicates that stochastic dynamics might be at play in the climate system. This may lead in the end to a speculation about the predictability of climate.

\section{The radiative energy balance}

The climate system and the biosphere, for that sake, is driven by the balance of the incoming low entropic short wave radiation from the Sun and the outgoing high entropic long wave black-body radiation to space. The possibility of driving the climate and biological 'engines' comes from the big temperature differences between the surface of the Sun $(\sim 6000 \mathrm{~K})$, the surface of the Earth $(\sim 300 \mathrm{~K})$ and outer space $(\sim 3 \mathrm{~K})$. The surface temperature of the Earth depends on this balance. Firstly, the amount of incoming radiation depends on the planetary albedo. The albedo of an object is the fraction of sunlight hitting the object which is reflected, thus it is the complement to the absorption. The planetary albedo is not a constant factor, it depends through the amount of clouds and ice, on the state of the climate itself. The feedback of clouds on temperature is very complicated. It depends on the height in the atmosphere where the clouds are formed and the state of the atmosphere surrounding the clouds. The clouds cool by reflecting the incoming radiation and they heat by trapping the outgoing radiation. These are both strong factors, of equal magnitude, thus partially cancelling each other out, resulting in a net cooling effect. Ice and snow on the surface unambiguously cool by reflecting the incoming short wave radiation. The amount of ice and snow influences the planetary albedo. This effect we can describe in a model of the climate represented by just one parameter, the mean surface temperature $T$. We thus need to determine the functional relationship between this temperature and the long wave radiation and the albedo, respectively. The amount of ice and snow is larger when the temperature is lower, so the 
lower the temperature the higher the albedo, and we can write the albedo $\alpha(T)$ as a function of the temperature. If the temperature is below some low temperature $T_{1}$ the planet will be completely ice covered and a further decrease in temperature cannot increase the albedo. If the temperature is above some other high temperature $T_{2}$ the ice is completely melted and a further increase in temperature will not lead to a decrease in albedo. The simplest functional form is a linear dependence of the albedo on temperature in between these two temperatures. This is the most reasonable choice when no other information is available a priori. We then have the relation:

$$
\alpha(T)= \begin{cases}\alpha_{1}, & T \leq T_{1}, \\ \frac{\left(T_{2}-T\right) \alpha_{1}+\left(T-T_{1}\right) \alpha_{2}}{T_{2}-T_{1}}, & T_{1}<T \leq T_{2}, \\ \alpha_{2}, & T>T_{2} .\end{cases}
$$

The change of the temperature $T$ is determined by the difference in incoming and outgoing radiation $[1,2]$,

$$
c \frac{\mathrm{d} T}{\mathrm{~d} t}=R_{\mathrm{i}}-R_{\mathrm{o}}=(1-\alpha(T)) S^{*}-\sigma T_{\mathrm{eff}}^{4},
$$

where $c$ is the heat capacity, $S^{*}=S / 4$ is a quarter of the solar constant. (The quarter comes from the ratio of the surface of the sphere to the cross-sectional area blocking the sunlight.)

$T_{\text {eff }}$ is the temperature at the level in the atmosphere from where the black-body radiation is emitted to space. Since the atmosphere is opaque in the infrared wave-band due to greenhouse gas absorbers this level is estimated as one optical dept into the atmosphere in this band. The greenhouse effect, the change in cloudiness and other factors must all be expressed through the connection between the surface temperature $T$ and the temperature $T_{\text {eff }}$. The more greenhouse gas in the atmosphere the higher in altitude is the effective level of emission. The temperature decreases with height by convective adjustment, such that the atmosphere is stable with respect to vertical adiabatic motion. The greenhouse warming can then be defined as $\Delta T=T-T_{\text {eff }}$, such that the last term in Equation (2) becomes $R_{\mathrm{o}}=\sigma(T-\Delta T)^{4}$. The value of the planetary albedo can be obtained elegantly by comparing the bright and the dark side of the Moon at half Moon, the bright side is lit by the Sun, while the dark side is lit by sunlight reflected off the Earth. From this (and from satellite measurements) the planetary albedo is measured to be approximately 0.31 ; using this and solving (2) with respect to temperature we get the present-day greenhouse warming to be $\Delta T \approx 32 \mathrm{~K}$.

The greenhouse warming will in general be a function of $T$ mainly through the temperature dependent water vapour (a greenhouse gas) concentration and cloud formation.

The behaviour of (2) is easily understood from a graphic representation. Figure 2 shows the incoming and outgoing radiation as a function of temperature. There are three temperatures $T_{\mathrm{a}}, T_{\mathrm{b}}, T_{\mathrm{c}}$ for which the curves cross such that the incoming and outgoing radiations are in balance. These points are the fixed points of (2). Consider the climate to be at point $T_{a}$. If some small perturbation makes the temperature lower than $T_{a}$ we will have $R_{\mathrm{i}}>R_{\mathrm{o}} \Rightarrow c \mathrm{~d} T / \mathrm{d} t>0$ and the temperature will rise to $T_{a}$. If on the other hand, the perturbation is positive and the temperature is a small amount larger than $T_{a}$ we have $R_{\mathrm{i}}<R_{\mathrm{O}} \Rightarrow c \mathrm{~d} T /$ $\mathrm{d} t>0$ and the temperature will decrease to $T_{a}$ again. Thus, $T_{a}$ is a stable fixed point. The same analysis shows that for $T_{b}$ a small perturbation will grow in time and the temperature will move away from $T_{b}$. Thus, $T_{b}$ is an unstable fixed point. A similar analysis shows that $T_{c}$ is a stable fixed point. The tendencies are indicated by arrows in Figure 2. The model has two stable climate states $T_{a}$ and $T_{c}$. So if the temperature at some initial time is lower than $T_{b}$ it will eventually reach the temperature $T_{a}$ and if it is higher than $T_{b}$ it will reach the temperature $T_{c}$. The present climate is the climate state $T_{c}$ where the ice albedo does not play a significant role in cooling the Earth.

The radiation might not be constant in time but depends on some external factors. The solar flux varies both because of changes in solar output and because of changes in the Earth's inclination and orbit around the Sun. The solar constant can then be expressed as $\mu S^{*}$, where $\mu>0$ is a time dependent parameter so we have $R_{\mathrm{i}}=(1-\alpha) \mu S^{*}$ in (2). Consider the climate represented by $T_{c}$ in the situation $\mu>1$. Then for $\mu$ not too small, corresponding to the dashed curve in Figure 3,

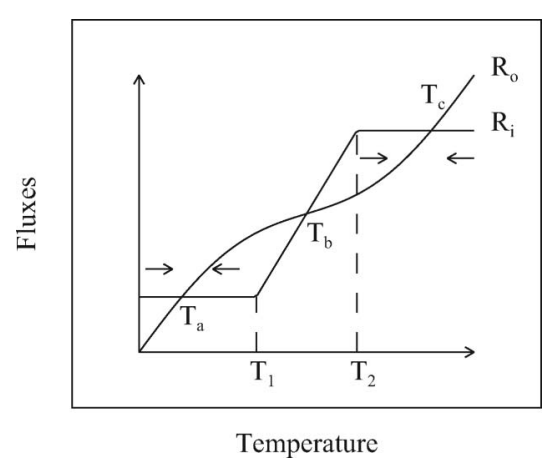

Figure 2. The global mean surface temperature is determined by the balance between incoming and outgoing radiation. The net incoming flux depends on temperature through the decreasing planetary albedo with temperature. The outgoing flux is determined by the black-body radiation and the greenhouse effect as a function of the surface temperature. 
the equilibrium temperature is lowered a little, as we would expect when the solar input decreases. However, if $\mu$ becomes smaller than some value $\mu_{0}$ the two curves do not cross anymore and there is no stable fixed point near $T_{c}$. The climate will then run into the only stable fixed point $T_{a}$ which is still present. A bifurcation has occurred and there is a large change in climate. This kind of behaviour is for obvious reasons sometimes also called catastrophic. If $\mu$ grows again, the climate state $T_{c}$ will not recover until $\mu$ exceeds some other value $\mu_{0}^{\prime}>1$, where $T_{a}$ disappears. This is the dotdashed curve. For each value of $\mu$ we have either one or three fixed points and we can plot the fixed points as a function of $\mu$. This is a bifurcation diagram (see Figure 4). The two full curves represent the stable fixed points and the middle dashed curve represents the unstable fixed point. As can also be seen from Figure 4 the unstable and one of the stable points coincide at the bifurcation points $\mu_{0}$ and $\mu_{0}^{\prime}$. Assuming that the solar flux changes periodically in time as $\mu(t)=\left(\mu_{0}+\mu_{0}^{\prime}\right) /$ $2+\varepsilon \sin \omega t$ where $\varepsilon>\left|\mu_{0}-\mu_{0}^{\prime}\right|$ the climate will jump periodically between the states $T_{a}$ and $T_{c}$. The evolution is represented by arrows in Figure 4, this is a hysteresis loop. If the climate $T(t)$ is considered as the response to the forcing function $\mu(t)$ the response is strongly nonlinear, which means that at some times there is a large response to a small change in forcing, which was originally motivating the model as an explanation for glacial cycles as a response to the weak forcing from the period changes in Earth orbit around the Sun. The time evolution is schematically shown in Figure 5.

\section{The Snowball Earth and the 'Faint young Sun paradox'}

The stable climate state $T_{a}$ corresponds to a totally ice covered planet and as such is not a realistic theory of the glacial cycles. The totally ice covered planet is the

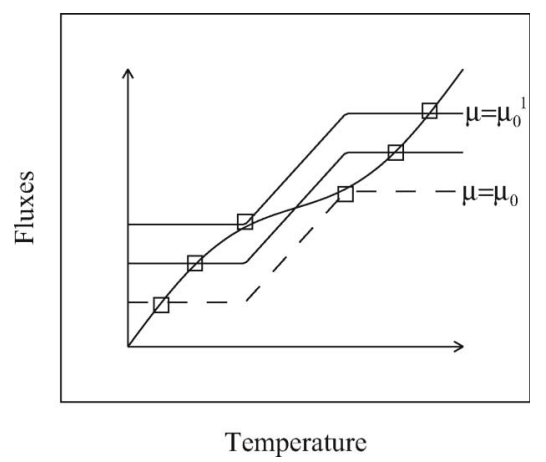

Figure 3. The incoming radiation change as a function of changes in the solar constant, represented by a control parameter $\mu$. For some larger value $\mu>\mu_{0}^{1}$ only the upper stable fixed point exists. For some smaller value $\mu<\mu$ only the lower stable fixed point exists.
Snowball Earth [3]. There is geological evidence of such an extreme 'deep freeze' climate several times in the Neoproterozoic period earlier than 700 million years ago. This is based on synchronous findings of glacial deposits like moraine in many places which at those times were near the equator. The speculated way out of the deep freeze is the following: the balance in geological timescales between weathering binding atmospheric $\mathrm{CO}_{2}$ into rocks and volcanic out-gassing of $\mathrm{CO}_{2}$ was changed during the deep freeze. Due to the cold conditions the atmosphere dried out and weathering was reduced. Unchanged volcanic out-gassing resulted in an ever increasing amount of $\mathrm{CO}_{2}$ in the atmosphere. At some point, after about 80 million years, this would result in a greenhouse warming strong enough to melt the ice. This scenario corresponds to gradually moving the $R_{\mathrm{o}}$ curve upwards in Figure 2 until the stable fixed point $T_{a}$ disappears (together with $T_{b}$ ) through a saddle-node bifurcation, and the only remaining fixed point is $T_{c}$. The warming would then be almost explosive with global mean temperatures going from some $-40^{\circ} \mathrm{C}$ to

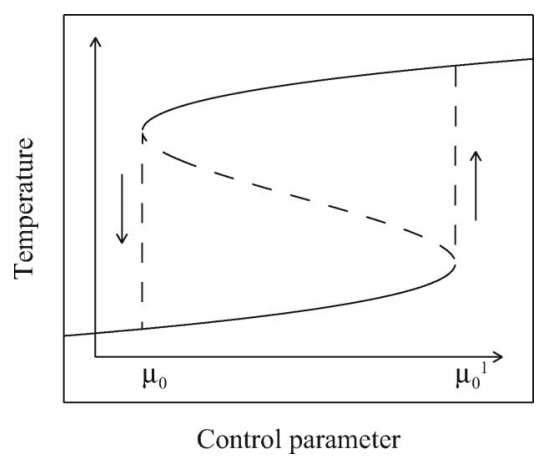

Figure 4. The fixed points plotted as a function of the control parameter $\mu$. At $\mu \nearrow \mu_{0}^{1}$ the lower state disappears through a saddle-node bifurcation, while at $\mu \searrow \mu_{0}$ the upper state disappears. A cyclic change in $\mu$ leads to a hysteresis loop.

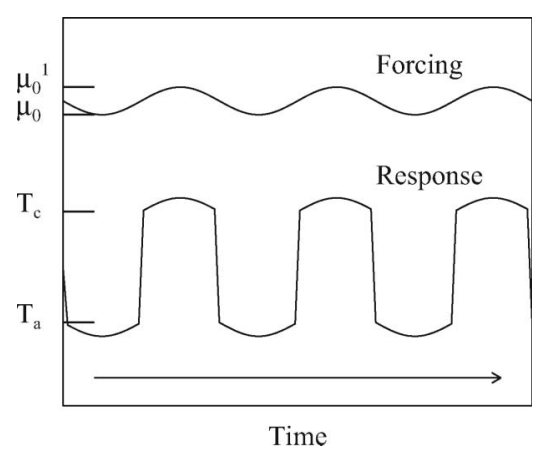

Figure 5. A small periodic variation in the forcing can lead to a strongly non-linear large response through a hysteresis loop. 
some $+50^{\circ} \mathrm{C}$ within a few years. Few organisms would be able to survive such a dramatic climatic jump, and we could speculate that this is a reason why it took such a long period for multicellular life to evolve. Multicellular life came into existence some 600 million years ago in the Cambrian explosion shortly after the last deep freeze. To substantiate such a speculation of course, unsuccessful development of multicellular life terminated by a Snowball Earth event must be found.

The fact that the Earth was not frozen in its very early history despite the fact that the Sun was about $30 \%$ fainter is known as the 'Faint young Sun paradox'. The most probable solution to the puzzle (which is not really a paradox) is that the geochemical carbon cycle was balancing the out-gassing of $\mathrm{CO}_{2}$ from volcanoes with $\mathrm{CO}_{2}$ removal through weathering, where carbonic acid rain slowly dissolves silicate rocks [4]. The balance between these two processes determines the atmospheric $\mathrm{CO}_{2}$ concentration and thus the temperature through greenhouse warming. Obviously, this can only work as a regulator of temperature if one of these processes depends on the temperature itself [5]. This is the case for weathering, since it depends strongly on precipitation. The regulation acts like this negative feedback loop: more atmospheric $\mathrm{CO}_{2} \rightarrow$ higher temperature $\rightarrow$ more rain $\rightarrow$ stronger weathering $\rightarrow$ less atmospheric $\mathrm{CO}_{2}$. The reason why Snowball Earth events have not happened since before the Cambrian explosion is thought to be a pole-ward continental drift changing the hydrological cycle and the weathering of atmospheric $\mathrm{CO}_{2}$.

\section{One-component dynamical systems}

Models like the Bodyko-Sellers climate model introduced above are very useful in the study of climate dynamics. It is therefore relevant to exploit the behaviour in mathematical terms in a more general setting. Consider the system as described by the variable $x$ by the equation

$$
\begin{gathered}
\dot{x}(t)=f(x(t), \mu), \\
x(0)=x_{\mathrm{i}},
\end{gathered}
$$

where as usual the dot means differentiation with respect to time and $\mu$ is some parameter called a control parameter. This is the simplest case where the system is described by only one dependent variable, so we can call the system a 'one-component' dynamical system. Equation (3) is an autonomous ( $f$ does not depend explicitly on time, disregarding for now any possible time dependence of $\mu$ ), first order (one differentiation with respect to time), ordinary (no partial derivatives) differential equation. It has a unique solution with the specified initial condition (4) where the subscript ' $i$ ' indicates 'initial'. For the moment we will suppress the dependence on the external parameter $\mu$. Equation (3) can be integrated,

$$
\frac{\mathrm{d} x}{\mathrm{~d} t}=f(x) \Rightarrow \int_{x_{i}}^{x(t)} \frac{\mathrm{d} x}{f(x)}=\int_{0}^{t} \mathrm{~d} t=t
$$

to obtain $x$ as an implicit function of $t$, the integrand is only defined for $f(x) \neq 0$. If $f\left(x_{0}\right)=0$ then $x_{0}$ will be a fixed point where the solution will reside and the integral above is not defined.

The fixed points of Equation (3) are determined as the set of solutions to the equation

$$
f(x)=0 .
$$

Having found a fixed point $x_{0}$ the next thing to do is to determine the stability of $x_{0}$. When $x$ is close to $x_{0}$ we can expand $f(x)$ in a Taylor series around $x_{0}: f(x)=$ $f\left(x_{0}\right)+f^{\prime}\left(x_{0}\right)\left(x-x_{0}\right)+\mathcal{O}\left(\left(x-x_{0}\right)^{2}\right)=f^{\prime}\left(x_{0}\right)\left(x-x_{0}\right)+$ $\mathcal{O}\left(\left(x-x_{0}\right)^{2}\right)$. Inserting this into (3), defining the small perturbation $y=x-x_{0}$ we get to first order,

$$
\dot{y}=f^{\prime}\left(x_{0}\right) y \Rightarrow y(t)=y(0) \exp \left(f^{\prime}\left(x_{0}\right) t\right) .
$$

If $f^{\prime}\left(x_{0}\right)>0$ the perturbation $y(0)$ will grow exponentially with time and the fixed point $x_{0}$ is unstable. If $f^{\prime}\left(x_{0}\right)<0$ the perturbation will decrease exponentially in time and the fixed point is stable, so the stability is determined by the first derivative $f^{\prime}\left(x_{0}\right)$ at the fixed point. The interval of initial conditions $x_{i}$ which eventually will end up in (or arbitrarily close to) the stable fixed point $x_{0}$ is called the domain of attraction of $x_{0}$. Consider $x_{1}<x_{2}$ to be two stable fixed points and assume that there are no other stable fixed points between $x_{1}$ and $x_{2}$. If $x_{1}<$ $x<x_{2}$ is a point close to $x_{1}$ then $x$ will approach $x_{1}$ with time and thus $f(x)<0$. On the other hand, if $x$ is close to $x_{2}$ it must approach $x_{2}$ with time and $f(x)>0$. Thus, there must exist a point $x_{3}$ separating the domains of attraction of $x_{1}$ and $x_{2}$ such that $x_{1}<x_{3}<x_{2}$ and $f\left(x_{3}\right)=0$. This fixed point must be unstable since we assumed that there were no stable fixed points between $x_{1}$ and $x_{2}$. This shows that any two stable fixed points must be separated by an unstable fixed point, and by a completely similar argument two unstable fixed points must be separated by a stable fixed point. Therefore, in the stability analysis of the climate model above it suffices to see that $T_{a}$ is a stable fixed point. From this it follows that $T_{b}$ must be unstable and $T_{c}$ must be stable. This analysis is easily understood if the equation of motion (3) is expressed in terms of a potential,

$$
\dot{x}=-\frac{\mathrm{d} U(x)}{\mathrm{d} x},
$$


where $U(x)=\int{ }^{x} f(y) \mathrm{d} y$ is the potential corresponding to the force $f(x)$. The fixed points correspond to local extrema for the potential. The stability of the fixed points are determined by the curvatures $f^{\prime}\left(x_{0}\right)=$ $-U^{\prime \prime}\left(x_{0}\right)$. So if $U^{\prime \prime}\left(x_{0}\right)>0$ then $U\left(x_{0}\right)$ is a minimum and $x_{0}$ is a stable fixed point. If $U^{\prime \prime}\left(x_{0}\right)<0$ then $U\left(x_{0}\right)$ is a maximum and $x_{0}$ is an unstable fixed point. Figure 6 shows the potential for (2) which is a double-well potential. It is now trivial, why two stable fixed points must be separated by an unstable fixed point.

\section{Stochastic climate dynamics}

The climate system obviously contains very many variables interacting in complex ways, so when the extreme reduction in representing only one variable governed by the energy balance we should consider Equation (2) as an effective equation and seek a way of incorporating the effect of the unresolved variables. The dynamics is characterised by the interaction between components with very different typical timescales. The atmospheric variations, weather patterns, are typically of days to weeks duration while variations in ocean currents are on much longer timescales, years to centuries, buildup of ice sheets takes several millennia and even changes in tectonics and geochemistry on millions of years timescales are influential in the climate.

The timescales where the slow climate variables change appreciably are beyond the correlation time for the fast variables. At these timescales the fast variables are effectively decorrelated and could then be described as being stochastic [6].

Assume that we can describe the system by a set of governing equations for the system variables that can be split in separate variables represented by the vectors

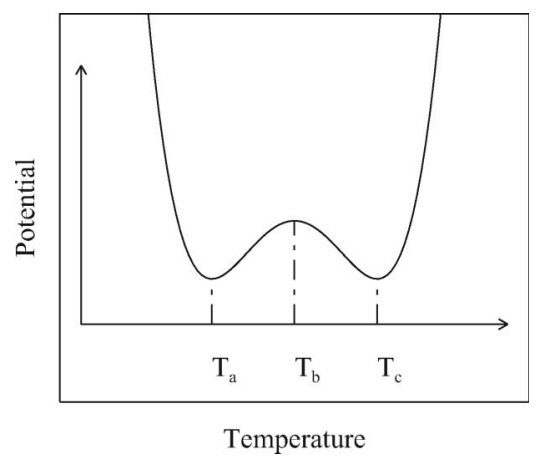

Figure 6. The double-well potential corresponding to the forcing in Equation (2). In this case $\mu_{0}<\mu<\mu_{1}$ such that both states with temperatures $\mathrm{T}_{a}$ and $T_{c}$ are stable. $T_{b}$ is the temperature of the separating unstable fixed point state represented by the dashed line in Figure 4. $x=\left(x_{1}, \ldots, x_{i}, \ldots\right)$ and $y=\left(y_{1}, \ldots, y_{j}, \ldots\right)$. Then the system differential equations can be written in terms of two sets of functions $f_{i}$ and $g_{j}$ as

$$
\begin{aligned}
& \dot{x}_{i}=f_{i}(x, y), \\
& \dot{y}_{j}=g_{j}(x, y),
\end{aligned}
$$

where we can associate a typical timescale $\tau_{x i}$ and $\tau_{y j}$, respectively, to each variable such that $\tau_{x i} \ll \tau_{y j}$ for all $(i, j)$. Equation (10) describes the dynamics of the large scale observable. For brevity we drop vector notations, and consider $x$ and $y$ as scalar variables. The extension to more dimensions is mostly straightforward. In the effective dynamics for $y$ we can write the small scale variable as $x=\langle x \mid y\rangle+x^{\prime}$, where the brackets denote the average of $x$ conditioned on $y$. Inserting this into the second equation, using that $x$ varies much faster than $y$, we can approximate

$$
\begin{aligned}
\dot{y} & =g\left(\langle x \mid y\rangle+x^{\prime}, y\right) \approx g(\langle x \mid y\rangle, y)+\partial_{x} g(\langle x \mid y\rangle, y) x^{\prime} \\
& =g_{\text {eff }}(y)+\sigma(y) \eta .
\end{aligned}
$$

For the short time correlated variations $x^{\prime}(t)$ we have substituted a stochastic white noise $\sigma(y) \eta(t)$ with $\left\langle\eta(t) \eta\left(t^{\prime}\right)\right\rangle=\delta\left(t-t^{\prime}\right)$.

Equation (11) is a Langevin equation. In the simplest form we will assume the noise intensity to be independent of the climate state $y$. Writing $F(y)$ for $g_{\text {eff }}(y)$ we have

$$
\dot{y}=F(y)+\sigma \eta=-\mathrm{d} U / \mathrm{d} y+\sigma \eta,
$$

where the drift 'force' $F(y)$ is defined as (minus) the gradient of an effective potential $U(y)$. If the noise intensity is low the $y$ component will for a long time settle around a stable fixed point $y_{0}$ provided $F\left(y_{0}\right)=0$ and $F^{\prime}\left(y_{0}\right)=-\alpha<0$. The notion of low noise intensity must be defined in the sense of an Arrhenius escape time, $T \sim \exp \left(h / \sigma^{2}\right)$, where $h$ is the barrier height in the potential $U$ separating the stable fixed point $y_{0}$ from other stable fixed points. Without loss of generality we can take $y_{0}=0$ and expand $F(y)$ in (12) to first order

$$
\dot{y}=-\alpha y+\sigma \eta \text {. }
$$

A process satisfying this linear stochastic equation is called an Ornstein-Uhlenbeck process or a red noise process [7]. Equation (13) can easily be solved for the auto-correlation function, $c(\tau)=\langle y(t) y(t+\tau)\rangle$ :

$$
c(\tau)=\left\langle y^{2}\right\rangle \exp (-\alpha|\tau|),
$$


where the correlation time is $T=1 / \alpha$. The powerspectrum is the Fourier transform of the autocorrelation function, which becomes,

$$
P(\omega)=\sigma^{2} /\left(\omega^{2}+\alpha^{2}\right),
$$

and the connection between the intensity of the process and the intensity of the noise is $\left\langle y^{2}\right\rangle=\sigma^{2} /(2 \alpha)$. This is the simplest form of the fluctuation-dissipation theorem.

The red noise process (13) is quite a good model of the variations of climate series in our present climate. Figure 7 shows the power spectrum of the last 4000 years of a Greenland ice-core proxy record [8] of temperature. The smooth curve is given by Equation (14) with a correlation time of 5 years. The peak, indicated by the arrow is the annual cycle in temperature, which is not included in the simple description (13). The correlation time of 5 years should be expected from the typical timescales of variations in surface water temperatures in the North Atlantic ocean influencing the air temperatures in that region.

The Langevin Equations (11) or (12) are stochastic and will as such have a solution which depends not only on the initial condition $y_{0}\left(t_{0}\right)$, it will also depend on the specific realisation of the noise. A deterministic equation for the conditional probability $p\left(x, t \mid x_{0}, t_{0}\right)$, which is the probability density for $x$ at time $t$, given

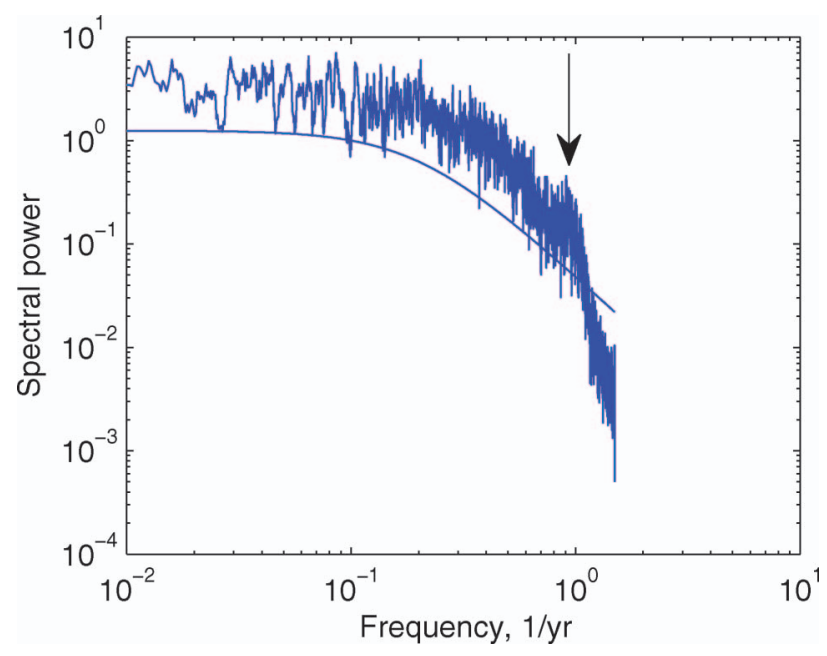

Figure 7. The power-spectrum of the last 4000 years of a Greenland ice-core proxy record of temperature (Dansgaard et al. [8]). The smooth curve is given by Equation (14) with a correlation time of 5 years. The peak, indicated by the arrow, is the annual cycle in temperature. The annual cycle is not included in the red noise process (13). the process is at $x_{0}$ at time $t_{0}$, can be derived from Equation (12) [7]:

$$
\begin{aligned}
\partial_{t} p\left(x, t \mid x_{0}, t_{0}\right)=- & \partial_{x}\left[F(x) p\left(x, t \mid x_{0}, t_{0}\right)\right] \\
& +\partial_{x}^{2}\left[\frac{\sigma^{2}(x)}{2} p\left(x, t \mid x_{0}, t_{0}\right)\right] .
\end{aligned}
$$

This is the Fokker-Planck equation for the conditional probability density.

\section{Ice ages}

The climate has varied periodically between ice ages and warm periods for the last few tens of millions of years. A continuous record of these variations is found in sedimentation in the deep sea. The sediments are formed from sinking planktonic organisms (foramenifera). The deep sea is biologically a desert, thus the sedimentation rate is extremely low, of the order of a metre in a million years. The oxygen isotopes in the calcium-carbonate shells of these organisms is a proxy for the isotopic composition of the ocean water, and since light isotopes evaporate easier a proxy for the amount of water deposited in ice sheets. A composite of deep sea records is shown in Figure 8 [9]. Since going deeper into the sediments corresponds to going back in time, the geological convention is to invert the time axis. So keep in mind that time grows from right to left in the figure. The convention for the units 'years BP' is 'year before present', with 'present' $=1950$. The graph should be read as a proxy-temperature record, showing cycles of warm (high values) and cold periods (low values).

The glacial cycles are attributed to the climatic response of the orbital changes in the irradiance to the Earth $[10,11]$. These changes in the forcing are too small to explain the observed climate variations as simple linear responses. Non-linear amplifications are necessary to account for the glacial cycles. The dominant orbital periods in solar insolation is the 41 kyr obliquity cycle (tilt of rotational axis, determining the meridional gradient in insolation) and the precessional cycles (determining the season when Earth is closest to the Sun) which decompose into $19 \mathrm{kyr}$ and $23 \mathrm{kyr}$ periods. However, through the last $800 \mathrm{kyr}-$ $1 \mathrm{Myr}$ the dominant period for the glacial cycles is approximately $100 \mathrm{kyr}$ similar to the one order of magnitude weaker eccentricity cycle (determining the semi-annual difference in distance to the Sun). The weakness of this climatic forcing is referred to as the $100 \mathrm{kyr}$ problem of the Milankovitch theory [12]. It is now generally accepted that the $100 \mathrm{kyr}$ glacial timescale cannot be attributed to the eccentricity cycle [9]. In the Plio- and early Pleistocene, 3-1 Myr BP, the 


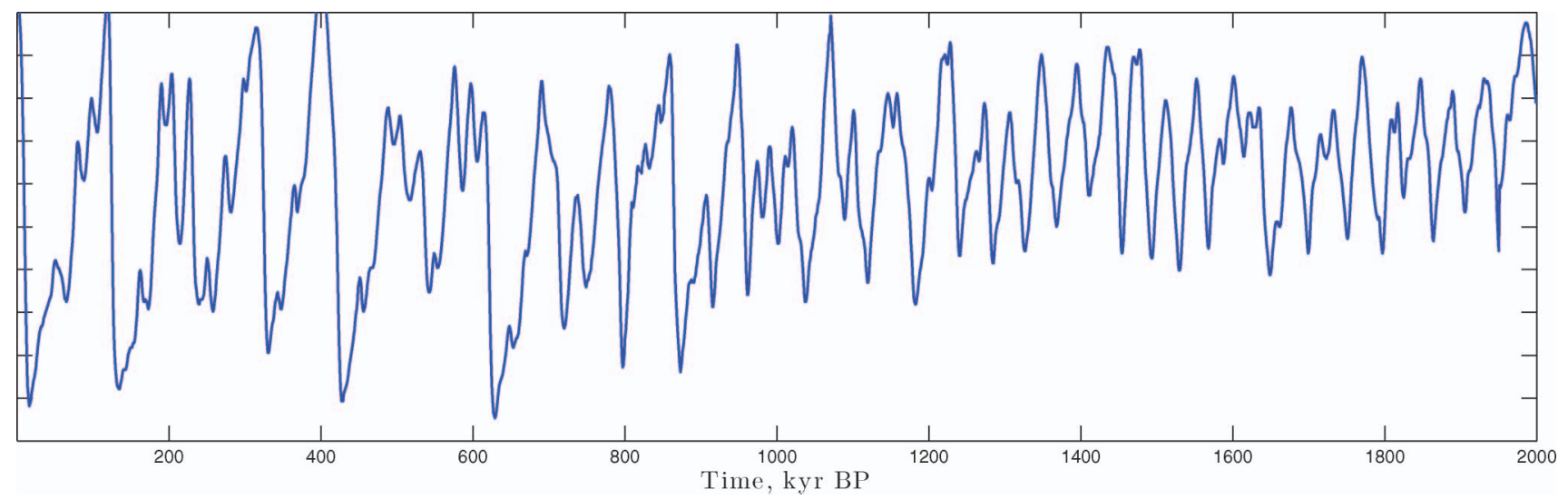

Figure 8. The (normalised) paleoclimatic isotope record from a composite of ocean cores. The record is a proxy for the global sea level or minus the global ice volume. The curve shows changes between ice ages and warm periods through the last two million years. Note that the geological convention of time increasing from right to left is followed, thus present time is at the origin.

dominant period of variation was indeed the $41 \mathrm{kyr}$ obliquity variation $[13,14]$.

Different mechanisms have been proposed to explain the occurrence of the $100 \mathrm{kyr}$ glacial cycle. These range from self-sustained non-linear oscillators [15-17], forced non-linear oscillators [18] to the glaciations being a resonance phenomenon. Stochastic resonance, which we shall return to later, was originally constructed as an explanation of ice ages [19].

Combined evidence from records of glaciations on land and deep sea records suggest that the climate has shifted between different quasi-stable states characterised by the mode of the global ocean circulation and the degree of glaciation [20]. These states may originate similar to the stable states in the simple ocean box model which we shall introduce later, or the energy balance model introduced above. By comparison between the paleoclimatic record and a non-linear stochastic model, it is demonstrated that the record can be generated by the forcing from insolation changes mainly due to the obliquity cycle through the full record including the last $1 \mathrm{Myr}$. It has been long known that the ' $100 \mathrm{kyr}$ world' is not linearly responding to the orbital forcing [21], but even in the ' $41 \mathrm{kyr}$ world' the climate response to the orbital forcing is non-linear [22]. The assumption here is that the orbital forcing resulted in periodic jumps between two stable climate states. What happened approximately $800 \mathrm{kyr}-1 \mathrm{Myr}$ ago was that a third deep glacial state became accessible resulting in a change in length of the glacial cycles. The reason for this midPleistocene transition (MPT) is unknown, and attributed to a gradual cooling due to a decreasing atmospheric $\mathrm{CO}_{2}$ level [23] or a change in the bedrock erosion (the regolith hypothesis) [24].
Since in the energy balance model (2) of the Earth, the climate is characterised by only one variable, the global mean temperature, there is ambiguity in ascribing the orbital forcing from the time and space varying insolation field across the globe.

Huybers et al. $[13,25]$ argue that the ice melt-off depends on the integrated summer insolation indicating that this could be the relevant measure of orbital forcing $[13,25]$. This is closely related to the concept of degree days, which is the annual number of days with temperatures above freezing. This measure is dominated by the obliquity cycle since the increased insolation when Earth is close to the Sun in its orbit is compensated by shorter time spent there due to Kepler's second law. Thus the total insolation during the degree days becomes independent of the precessional cycle.

In contrast to this, Paillard [26] shows using a simple rule-based model of jumping between three different quasi-stationary climate states, that the climate record can be a response to the summer solstice insolation at $65 \mathrm{~N}$. The two proposed forcings (degree day insolation and June 26 insolation) are different, since the latter has a strong component of the precessional cycle. Using the June 26 insolation as the better proxy for the forcing can be rationalised from the point of view of a threshold crossing dynamics, since the extremal values (mid-summer insolation) would then be the governing parameter. However, since we cannot decide between the two within the framework of a simple model, we can take the alternative approach of assuming the linear combination of the two, considered as a first order expansion, which gives the best fit for the observed record as a response to the forcing. 


\section{The non-linear stochastic ice age model}

Due to the high dimensionality and the stochastic nature of the climate fluctuations it is highly unlikely that regular periodicities can result from internal oscillatory modes alone. It is much more plausible that non-linear responses to weak external periodic forcing would lead to periodic behaviour. There is evidence from observations as well as models that multiple states exist in the climate system [20]. This suggests a possible scenario of periodically induced destabilisations of three quasi-stable climate states [27].

The three states can be identified with the ones labelled $G$ (deep glacial), $g$ (pre-glacial), and $i$ (interglacial), respectively, by Paillard [26]. Paillard observed in the paleoclimatic record that there seems to be 'forbidden' transitions between the three states. In the period 2-1 Myr BP the record shows regular oscillations between only the two states $i$ and $g$, while in the period $1-0 \mathrm{Myr} \mathrm{BP}$ there is only a specific sequence of occurrences: $i \rightarrow g \rightarrow G \rightarrow i$ permitted. The underlying bifurcation structure provides a dynamical explanation of this observation.

From the observed climate record we derive an empirical model. This assumes that the climate dynamics are reflected in a single variable $x(t)$, which is taken to be the global mean surface temperature anomaly represented by the deep sea oxygen isotope proxy record shown in Figure 8. The dynamics are described by an effective non-linear stochastic differential equation,

$$
\dot{x}=f_{\alpha}(x, \mu)+\sigma \eta,
$$

where the white noise term $\eta$ with intensity $\sigma$ describes the influence of the non-resolved variables and the internally generated chaotic climate fluctuations. It is within this framework that the roles of the orbital forcing and internal stochastic forcing are investigated. The deterministic part, $f_{\alpha}(x, \mu)$, of the dynamics depend on the external orbital forcing, labelled by a single control parameter $\mu$ and internal parameters, represented by $\alpha$. Note that Equation (16) is a nonautonomous generalisation of the Equation (12), since $\mu$ and $\alpha$ are time-dependent.

The full climate dynamics can obviously not be completely reconstructed by such a single valued function. However, since stability and bifurcations are topological quantities it could be robust with respect to the detailed dynamics modelled. It is thus the bifurcation structure of $f_{\alpha}(x, \mu)$, with respect to the control parameter $\mu$, which determines the climate development.

Guided by the observed record and the transition rules we can empirically construct a bifurcation diagram: Figure $9(a)$, shows the bifurcation diagram for the drift function $f_{\alpha}(x, \mu)$ as a function of $\mu$ at the time interval 2-1 Myr BP. The bifurcation diagram shows the curves $\left\{x_{0}(\mu) \mid f\left(x_{0}, \mu\right)=0\right\}$. The fat curves are the stable fixed point curves for which $\partial_{x} f<0$, while the thin curves are the unstable fixed point curves for which $\partial_{x} f>0$. Thus, in the case of no additional noise ( $\sigma=0$ in Equation (16)) the state of the system $x(t)$ is uniquely determined from the initial state $x(0)$ and the development of the forcing $\mu(t)$.

In the real climate system the internal noise is substantial and the system will not reside exactly in the
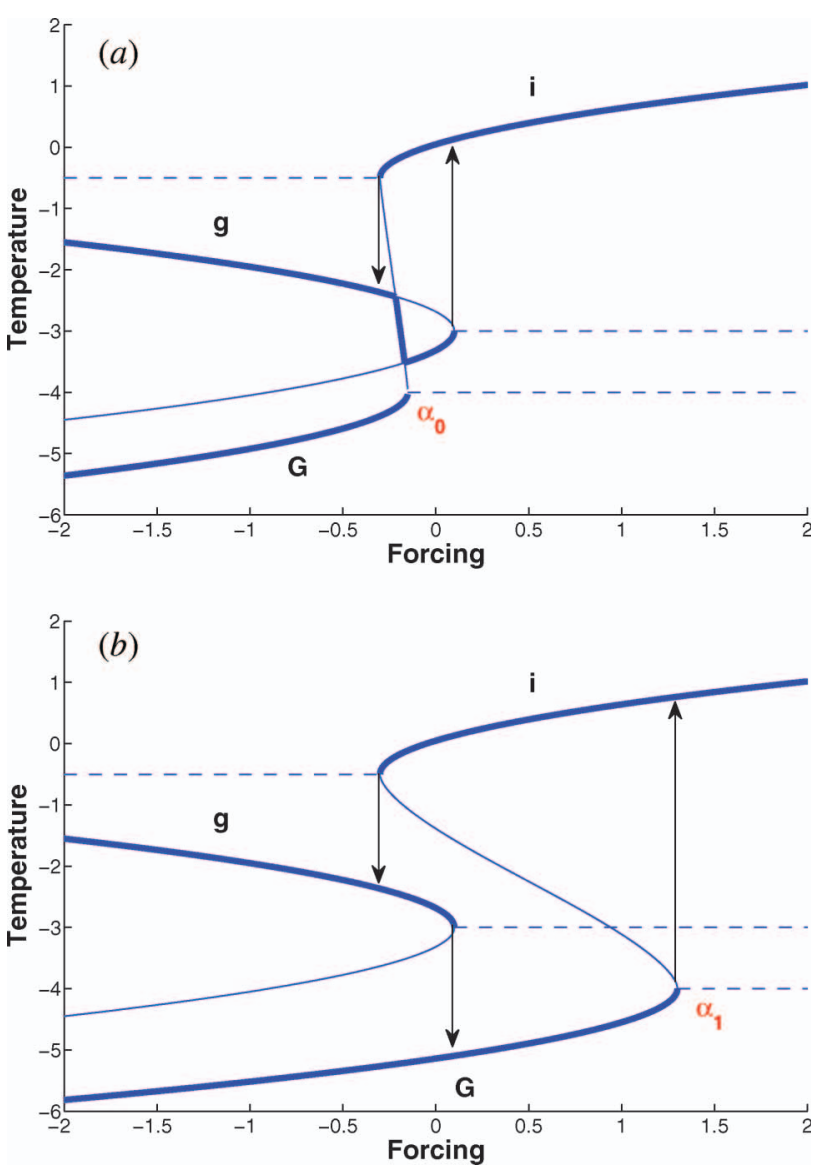

Figure 9. The bifurcation diagram for the ice age model. Along the $x$-axis is the forcing represented by the control parameter $\mu$, along the $y$-axis are the fixed points $\left\{x_{0}(\mu) \mid f\left(x_{0}, \mu\right)=0\right\}$ of the drift function $f_{\alpha}(x, \mu)$. The drift function is simply approximated by a fifth order polynomial, with the roots determined by the fixed points. The horizontal dashed line segments indicate (real part of) sets of complex conjugate roots. The fat curves show the stable fixed points. The bifurcation point $\alpha$ is the point where the deep glacial state $G$ disappears. The arrows indicate the hysteresis loop as the forcing parameter is changed. Upper panel: the glacial state $G$ is not accessible. Lower panel: now the location of the bifurcation point $\alpha$ has changed in such a way that the deep glacial state $G$ is accessible. 
steady states determined by the bifurcation diagram. Thus, the full drift function needs to be parameterised. The simplest way to parameterise the drift function in accordance with the bifurcation diagram is as a fifth order polynomial:

$$
f_{\alpha}(x, \mu)=\prod_{j=1}^{5}\left(x-x_{\alpha}^{j}(\mu)\right),
$$

where $x_{\alpha}^{j}(\mu)$ is the $j$ th steady state (zero-points) in the bifurcation diagram. As labelled in the figure the parameter $\alpha$ determines the position of the lower bifurcation point.

It should be noted that this is of course not the only possible drift function corresponding to this bifurcation diagram. In order to reconstruct the drift function from the observed realisation, one could in principle obtain the stationary probability density $p_{\mu 0}(x)$ by sorting $x(t)$ according to $\mu(t)=\mu_{0}$. Assuming that $\mu(t)$ is changing slowly in comparison to the timescale for $x(t)$ to drift to a stationary state $x_{0}\left(f_{\alpha}\left(x_{0}, \mu\right)=0\right)$, one could then obtain $f_{\alpha}\left(x, \mu_{0}\right)$ by solving the FokkerPlanck Equation (15) associated with Equation (16) for fixed $\mu=\mu_{0}$. This would require a very long data series and complete absence of additional non-climatic noise in the proxy data. This is not the case for the existing paleoclimatic record.

The climate forcing is, as mentioned before, taken to be a linear combination of the summer solstice $65 \mathrm{~N}$ insolation $\left(f_{\mathrm{ss}}\right)$ and the integrated summer insolation at $65 \mathrm{~N}\left(\bar{f}_{I}\right)$, where the summer period is defined as the period where the daily mean insolation exceeds $I=200 \mathrm{~W} \mathrm{~m}^{-2}$. The model results are robust with respect to the threshold $I$ chosen in a rather broad interval. The forcing, $f=\lambda \bar{f}_{I}+(1-\lambda) f_{\text {ss }}$, is shown in Figure 11, second panel, in the next section [28]. Values of $\lambda$ around 0.5 gives the best result, $\lambda=0.5$ is used. This assignment might, within the framework of the non-linear model, be interpreted as an empirical determination of the dominating components of the orbital forcing.

\section{The hysteresis behaviour}

The diagram in Figure 9(a), shows the fixed points of $f_{\alpha}(x, \mu)$ as a function of the deterministic forcing $\mu$. The three branches of stable fixed points $x^{j}(\mu)$ for the function, such that $f_{\alpha}\left(x^{j}, \mu\right)=0$ and $\partial_{x} f_{\alpha}\left(x^{j}, \mu\right)<0$, are indicated by fat curves. The specification of the $x^{j}(\mu)$ 's and Equations (16) and (17) completely defines the model, see [27] for more details. Since $x$ is a proxy for the global mean surface temperature anomaly, or minus the global ice volume, the lower branch corresponds to the deep glacial state $G$. The middle branch corresponds to the climate state $g$ and the upper branch to the interglacial state $i$. The thin curves correspond to the separating unstable fixed points. The dashed line-segments correspond to pairs of complex conjugate roots in the fifth order polynomial. Note again that assuming a polynomial drift function, this is uniquely determined from the roots, except from a trivial multiplicative constant.

Suppose that the climate is in either of the states $g$ or $i$ and the climatic noise is too weak to induce a crossing of a barrier separating the stable states. Then the only way a forcing induced shift between the climate states can occur is through bifurcations and a hysteresis loop $i \rightarrow g \rightarrow i$ as sketched by the arrows. Clearly the climate state $G$ is unreachable.

Assume now that the lower bifurcation point, indicated by $\alpha_{0}$ in Figure 9(a), moves toward larger values of $\mu$ indicating that a stronger forcing is needed in order to destabilise the deep glacial state. In this case, $\alpha_{0} \rightarrow \alpha_{1}$ shown in Figure $9(b)$, the glacial state $G$ is now reachable and a hysteresis loop $i \rightarrow g \rightarrow G \rightarrow i$ will appear. The central postulate of the model is the change in this bifurcation structure represented by the shift of the point $\alpha$ (from $\alpha_{0}$ to $\alpha_{1}$ on the $\mu$-axis) at the mid-Pleistocene transition. This constitutes a dynamical explanation for the 'rules of forbidden transitions' which are apparently obeyed by the observed record.

The change in the position of the lower bifurcation point is modelled such that $\alpha=\alpha_{1}$ when the climate is in state $i$. When the state $G$ is reached through two bifurcations, $\alpha$ is gradually changing. The gradual change in the bifurcation diagram is modelled as a relaxation, $\mathrm{d} \alpha / \mathrm{d} t=-\left(\alpha-\alpha_{0}\right) / \tau$, where $\alpha_{0}$ is the early Pleistocene equilibrium value and $\tau$ is a relaxation time. When the climate bifurcates through the rapid transition $G \rightarrow i$, the parameter $\alpha$ again changes to $\alpha_{1}$.

In order for the climate to skip the $41 \mathrm{kyr}$ obliquity pacing of deglaciations the timescale $\tau$ governing the bifurcation structure must be considerably longer than $41 \mathrm{kyr}$. The model results are quite insensitive to the specific value of $\tau$ in the interval $70-130 \mathrm{kyr}$, and is set to be $100 \mathrm{kyr}$. It is a major challenge to interpret the behaviour of the bifurcation point $\alpha$, governed by such a long timescale in terms of real climate dynamics. One could speculate that it is linked to the carbon cycle or with erosion of continents on these long timescales.

The presence of the stochastic forcing implies that the climate evolution is not fully deterministic. Figure 10, first panel, shows a particular realisation of the model. The second panel shows the forcing, the red curve shows the value of $\alpha$, which is defined as the position of the lower bifurcation point in Figure 9 along the axis of the forcing (the $x$-axis). Note that a transition $G \rightarrow i$ without noise assistance is only 

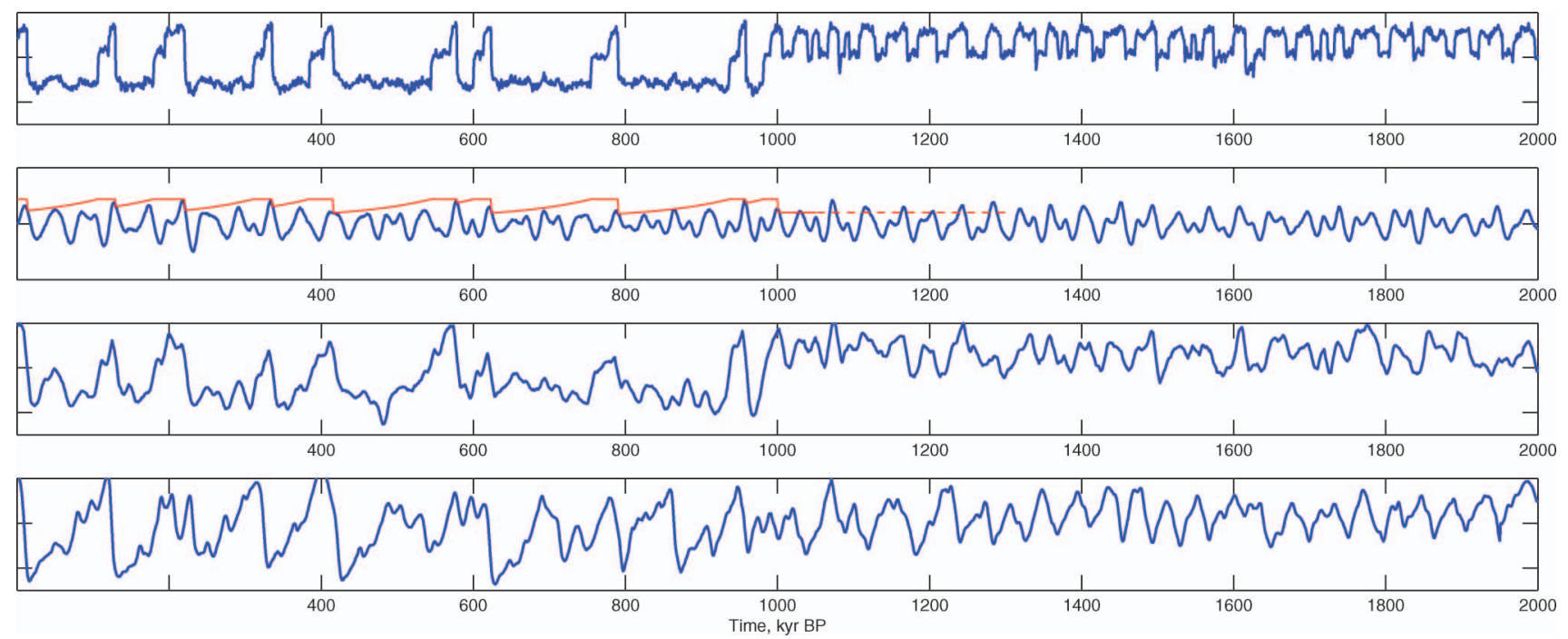

Figure 10. The top panel shows a realisation of the model. Second panel shows the orbital forcing driving the model. The red curve shows $\alpha(t)$, where the jumps to $\alpha=\alpha_{1}$ are triggered by the transition $G \rightarrow i$. The next transition is in the low noise limit, only possible when the blue curve is above the red curve. The third panel shows a 'pseudo paleorecord', where a red noise component representing the non-climatic noise, is added to the model realisation in the top panel. Lower panel is the same record as in Figure 8 for comparison.

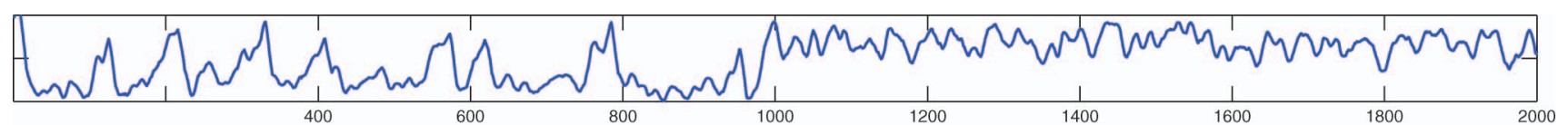

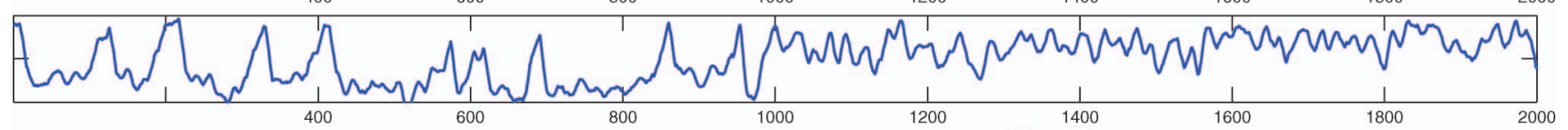

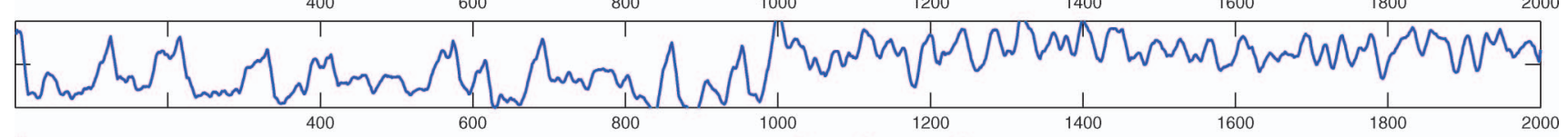

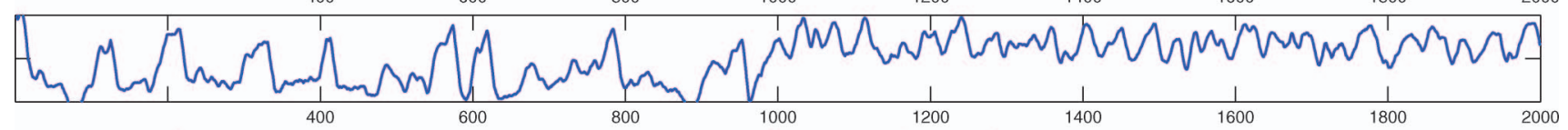

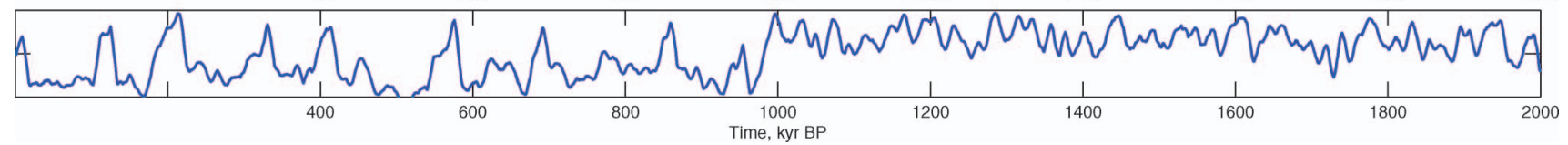

Figure 11. Five realisations of the model with the same orbital forcing and different stochastic forcing. The first panel shows a realisation without stochastic forcing. This is the purely deterministic climate response to the orbital forcing. The bottom four realisations have a noise intensity $\sigma=0.8 \mathrm{~K} \mathrm{kyr}^{-1 / 2}$. It is seen that only in the last part of the $100 \mathrm{kyr}$ world the timing of the terminations are independent from the noise. In all five realisations an additional non-climatic 'proxy noise' is added a posteriori.

possible when the forcing exceeds the value of $\alpha$ (that is when the blue curve is above the red curve in the second panel).

The composite Atlantic ocean sedimentation record in Figure 8 is repeated in the bottom panel for comparison. The curve is plotted with normalised variance and the mean subtracted. The dating of the record is based on a depth-age model independent from astronomical tuning.

The differences between the single records comprising the stacked record gives an estimate of the additional noise from bioturbation and other factors that make the record different from a true record of ice volume. So in order to compare the model with the observed climate 
record an additional red noise, of the same magnitude as the difference in deep sea records is added to the model. This is shown in the third panel, which should be compared with the observed record in the fourth panel.

In order to investigate the role of the stochastic noise, a set of realisations of the model is presented in Figure 11. For the comparison between the model and the proxy climate records we focus on the rapid transitions $G \rightarrow i$, called terminations [29,30]. The top panel shows a realisation with no stochastic noise. This is the deterministic climate response to the orbital forcing. It is seen that the last five terminations are reproduced as observed, but there are fewer interglacials in the earlier part of the late-Pleisocene period (1000-500 kyr BP) than in the observed record. From Figure 10, second panel, it is seen that the amplitude of the orbital forcing is low in this period. The bottom four panels show different realisations with a moderate stochastic forcing $\left(\sigma=0.8 \mathrm{~K} \mathrm{kyr}^{-1 / 2}\right)$. In these realisations the added noise induces additional terminations at different times, suggesting a fundamental unpredictability in glacial terminations.

\section{Noise induced transitions within the last glacial period}

The last glacial period showed millennium scale climatic shifts between two different stable climate states. The state of thermohaline ocean circulation probably governs the climate, and the triggering mechanism for climate changes is random fluctuations of the atmospheric forcing on the ocean circulation.

The high temporal resolution paleoclimatic data from ice cores are consistent with this picture and a bistable climate pseudo-potential can be derived. It is found that the fast timescale noise forcing the climate contains a component with an $\alpha$-stable distribution, also called a Levy flight [31]. As a radical consequence the abrupt climatic changes observed could in principle be triggered by single extreme events. These events are related to ocean-atmosphere dynamics on annual or shorter timescales and could indicate a fundamental limitation in predictability of climate changes.

Paleoclimatic records from ice cores [8] show that the climate of the last glacial period experienced rapid transitions between two climatic states, the cold glacial periods and the warmer interstadials (DansgaardOeschger (DO) events). Deep sea sediment cores [32] and coral records [33] indicate that the ocean circulation is a key player in these climatic oscillations [34]. Ocean circulation models, from the most simple Stommel type [35] to the complex circulation models [36] show that different flow states can exist as stable climatic states. To show this we shall shortly divert into the simplest possible ocean model.

\section{The thermohaline circulation}

The large scale ocean circulation is as important as the atmospheric circulation for redistribution of heat on the planet. This is part of the cause for the climate belts not simply following the latitudes. The climate belts are traditionally defined by the occurrence of different species of tropical, subtropical, temperate or arctic plants. The ocean circulations are driven by shear from the atmospheric winds, from tidal forces and by gravity through the buoyancy of the water itself [37]. These are in turn 'the wind driven circulations', 'tides' and 'the thermohaline circulation'. The thermohaline circulation is driven by the sinking of heavy water and the rising of light water. The density of ocean water depends on two parameters; temperature $T$ and salinity $S$ : warmer water is lighter and saltier water is heavier. The density can then, to first order in the difference from the mean state, represented by $\left(T_{0}, S_{0}\right)$, be written as

$$
\begin{aligned}
\rho(T, S)= & \rho\left(T_{0}, S_{0}\right)+\partial_{T} \rho\left(T_{0}, S_{0}\right)\left(T-T_{0}\right) \\
& +\partial_{S} \rho\left(T_{0}, S_{0}\right)\left(S-S_{0}\right) \\
= & \rho_{0}(1-\alpha \Delta T+\beta \Delta S),
\end{aligned}
$$

where we have introduced the coefficients of expansion $-\alpha \rho_{0}, \beta \rho_{0}$ with respect to temperature and salinity [38]. The Stommel model is the simplest possible non-trivial model describing the dynamics of the thermohaline circulation [39]. We assume that the ocean can be split into two boxes $A$ and $B$, each ascribed a temperature and a salinity $T_{A}, S_{A}$ and $T_{B}$, $S_{B}$, respectively. These could be seen as mean quantities like $T_{A}=\int_{A} \mathrm{~d} x T(x)$. The boxes are connected by an upper and a lower hydraulic link, each from continuity carrying the same current $q$ in opposite directions, see Figure 12. The current will be driven by buoyancy and will depend on the density difference between the boxes. Heavy water will be transported in the lower link from the box containing the heavier water to the box containing the lighter water and vice versa for the upper link. For simplicity we can assume a linear relationship,

$$
q=C\left(\rho_{A}-\rho_{B}\right)=C \rho_{0}\left[\beta\left(S_{A}-S_{B}\right)-\alpha\left(T_{A}-T_{B}\right)\right],
$$

where $C$ is an empirical constant. Here a variable, in this case $q$, depending on the whole fluid flow, temperature - and salinity fields not resolved in the model - is substituted by a functional relationship between variables in the model. This kind of modelling 
is termed a parameterisation. The governing equations for the model are

$$
\begin{aligned}
& V_{A} \dot{T}_{A}=|q|\left(T_{B}-T_{A}\right)+\kappa_{T}\left(\tilde{T}_{A}-T_{A}\right), \\
& V_{B} \dot{T}_{B}=|q|\left(T_{A}-T_{B}\right)+\kappa_{T}\left(\tilde{T}_{B}-T_{B}\right), \\
& V_{A} \dot{S}_{A}=|q|\left(S_{B}-S_{A}\right)+\kappa_{S}\left(\tilde{S}_{A}-S_{A}\right), \\
& V_{B} \dot{S}_{B}=|q|\left(S_{A}-S_{B}\right)+\kappa_{S}\left(\tilde{S}_{B}-S_{B}\right) .
\end{aligned}
$$

Here $V_{A}$ and $V_{B}$ are the volumes of the two basins, which we for simplicity will regard as having same size $V_{A}=V_{B}=1$ and neglect all together. The first terms on the right-hand sides are simply the exchanges of water between the boxes, while the second terms parameterise all other forces restoring to some mean temperatures $\tilde{T}_{A}, \tilde{T}_{B}$ and salinities $\tilde{S}_{A}, \tilde{S}_{B}$, respectively. This latter parameterisation is termed a Newtonian cooling. The parameters $\kappa_{T}$ and $\kappa_{S}$ can be regarded as linear response coefficients. They signify the (inverse) timescales associated with restoring to the mean states from perturbations in temperatures or salinities. The four equations in (20) are linearly dependent: by introducing $T=T_{A}-T_{B}$ and $S=S_{A}-S_{B}$, subtracting the second equation from the first and the fourth from the third we get

$$
\begin{aligned}
& \dot{T}=-|q| T+\kappa_{T}(\tilde{T}-T), \\
& \dot{S}=-|q| S+\kappa_{S}(\tilde{S}-S),
\end{aligned}
$$

with $q=(\beta S-\alpha T)$. The constant $C$ is trivially eliminated by rescaling of time, $\kappa_{T}$ and $\kappa_{S}$. For a further reduction of notation we introduce $x=\alpha T$ and $y=\beta S$ so (21) and (22) become,

$$
\begin{aligned}
& \dot{x}=-|y-x| x+\kappa_{T}(\tilde{x}-x), \\
& \dot{y}=-|y-x| y+\kappa_{S}(\tilde{y}-y) .
\end{aligned}
$$

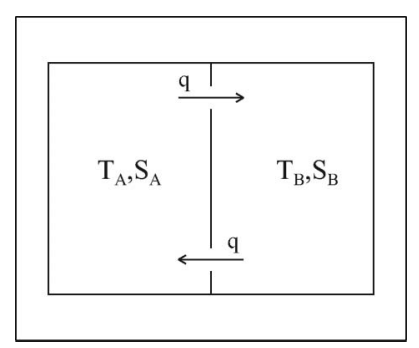

Figure 12. The Stommel model of the Atlantic ocean. The ocean is represented by two boxes: a warm tropical basin (A) and a cold extra-tropical and polar basin (B). These are connected by hydraulic links with a flux $q$, one in the surface ocean and one in the deep ocean. The state of the system is represented by the two mean temperatures in the boxes, $T_{A}$ and $T_{B}$ and the two mean salinities $S_{A}$ and $S_{B}$.
The forcing terms could be chosen in other ways, say by ascribing a differential heating $H$ to the temperature equation and a fresh water forcing $F$ to the salinity equation. The fresh water forcing represents the water evaporated from the warmer box transported by the atmosphere and precipitated into the colder box, leading to a net transport of salt in the opposite direction. The different forcings on the two equations are termed mixed boundary conditions. Had the forcings been the same: $\kappa_{T}=\kappa_{S}=\kappa$ the equations (23) and (24) could be further reduced. Introducing the variable $z=y-x$ :

$$
\dot{z}=-|z| z+\kappa(\tilde{z}-z)
$$

it is easy to see that this model has a single necessarily stable fixed point. So in order to observe any nontrivial behaviour it is crucial that the model has mixed boundary conditions. The mixed boundary conditions in the model are a natural consequence of the fact that the timescale for restoration of the salinity is much longer than the timescale for restoration of the temperature, $\kappa_{S} \ll \kappa_{T}$. The model has three fixed points which can be seen in the phase space portrait of the tendencies in Figure 13.

The phase space for a set of two coupled first order non-linear Equations such as (23) and (24) is very simple. Since trajectories cannot cross, most of the global information is obtained by identifying the fixed points. A short exhaustive list of possible fixed points is obtained from the linear stability of the fixed point. There will be two eigenvalues for the linearised system, assuming non-degeneracy of the eigen-space, these correspond to two perpendicular eigenvectors. If the eigenvalues are real, there are three possibilities: two negative eigenvalues corresponding to a stable fixed point, a negative and a positive eigenvalue corresponding to a saddle point and finally two positive eigenvalues corresponding to an unstable fixed point.

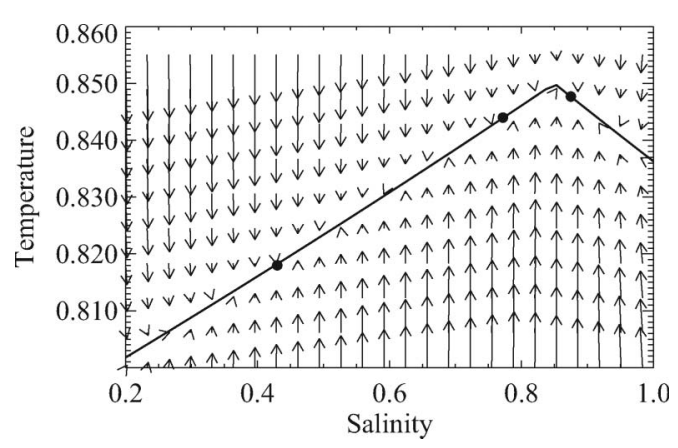

Figure 13. The phase space for the two-box Stommel model. The dots are the fixed points. As is seen from the phase space flow, temperature adjusts much faster than salinity. 
If the eigenvalues are complex this corresponds to stable, neutral or unstable foci, depending on the sign of the real part of the eigenvalues. The orbits along the eigenvectors, beginning and ending at fixed points, are the homoclinic (beginning and ending at the same (saddle) fixed point) and heteroclinic orbits (connecting stable and unstable fixed points or stable/unstable fixed points with saddle points). These orbits separate the phase space in basins of attraction for the stable fixed points. If an orbit is closed without passing through a fixed point it is a limit cycle.

In this case we can understand the behaviour of the model by assuming that temperature will equilibrate instantaneously, such that we can solve (23) in the case $\dot{x}=0$ and obtain the equilibrium temperature as a function of salinity. From this an effective potential depending on the salinity alone can be constructed by integrating the force along the curve $(S, T(S))$, as shown in Figure 14.

The changes in the thermohaline circulation of the Atlantic has probably been such that North Atlantic deep water (NADW) is produced in the warm periods and North Atlantic intermediate water in the cold periods $[40,41]$. The key question is then whether the switching between two stable climatic states of the oceanic flow can be internally triggered by the random forcing within the ocean-atmosphere system [42].

\section{Noise induced climate changes}

Taking the ice core record to be a climatic proxy resulting from the climate dynamics described through a Langevin equation of the type (12), we have to confirm that this is a consistent description and from the analysis to observe the structure of the noise driving the system. This provides strong constraints on the types of possible more realistic models of the underlying triggering mechanisms for the observed climatic shifts.

The calcium signal from the GRIP ice core is the highest temporal resolution glacial climate record

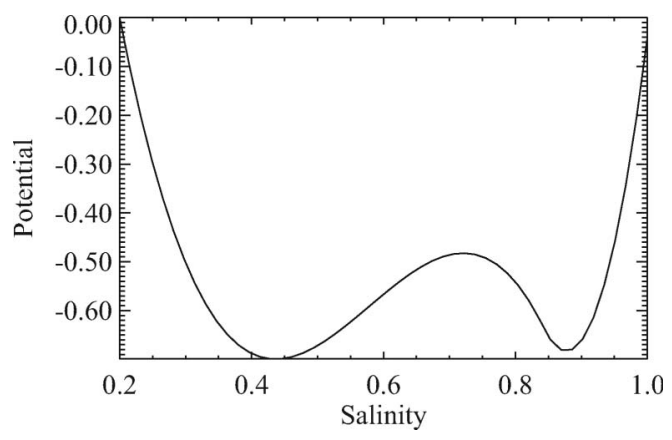

Figure 14. The effective potential $U(S)$ along the curve ( $S$, $T(S)$ ) shown as the fat curve in Figure 13. which exists [43]. The logarithm of the calcium signal is (negatively) correlated with the $\delta^{18} \mathrm{O}$ temperature proxy with a correlation coefficient of 0.8 [44], thus we use the logarithm of calcium as a climate proxy. This proxy has a very high temporal resolution, since it is related to dust in the ice and it is therefore not diffusing in the firn (the snow-pack before compactified to ice) as the $\delta^{18} \mathrm{O}$ isotope signal does. The temporal resolution of $\log (\mathrm{Ca})$ is about annual from $11 \mathrm{kyr}$ to $91 \mathrm{kyr}$ BP $(80,000$ data points). This is an order of magnitude higher than that of $\delta^{18} \mathrm{O}$. The calcium signal from the GRIP ice core is shown in Figure 15(a). The typical waiting time for jumping from one state to the other is between 1000 and 2000 years. The probability distribution for the waiting times between the beginning of the glacial - and the beginning of the following interstadial states - is shown in Figure 16. The straight line is an exponential distribution with mean waiting time of 1400 years. This is expressed as $P(T>t)=$ $\exp (-t / \tau)$, where $\tau=1400 \mathrm{yr}$ is the mean waiting time. The probability density function (PDF) of the signal, Figure 17, shows a bimodal distribution with peaks corresponding to the warm interstadials and the glacial state.

From the premise of the stochastic dynamics and the data we can now uniquely determine the climate pseudo-potential, $U(y)$, and the structure of the noise term. The noise term (diffusion term) is to first order, neglecting the drift term, defined as the derivative of the signal estimated as $\left(y_{t+\Delta t}-\mathrm{y}_{t}\right) / \Delta t$, shown in Figure $15(b)$. This signal is stationary except for a slow trend through the record which is partly due to smoothing with depth in the ice core. The intensity of the noise is thus approximately independent of the climate state itself.

The noise is approximately a white noise (not correlated in time) and has a strongly non-Gaussian distribution. Figure 18(a) shows the cumulated Probability on a scale on which a gaussian distribution is a straight line (probability paper scale). Figure 18(b) shows the two distribution tails on a log-log plot magnifying the behaviour of the tails. This has in an intermediate range a power function scaling with a power of about 2.75 and an additional extreme tail. The signal can only be described consistently by a Langevin equation if it is driven by two noise terms:

$$
\mathrm{d} y=-(\mathrm{d} U / \mathrm{d} y) \mathrm{d} t+\sigma_{1} \mathrm{~d} x+\sigma_{2} \mathrm{~d} L .
$$

The first noise component, $\sigma_{1} \mathrm{~d} x$, is generated by an additional Langevin equation, $\mathrm{d} x=-x \mathrm{~d} t+\left(1-x^{2}\right)^{1 / 2} \mathrm{~d} B$, where $x$ is an (unobserved) independent variable and $\mathrm{d} B$ is a unit variance Brownian noise. Here we have introduced the mathematically more correct notation of increments $\mathrm{d} y, \mathrm{~d} B$ etc. The notation is more correct 

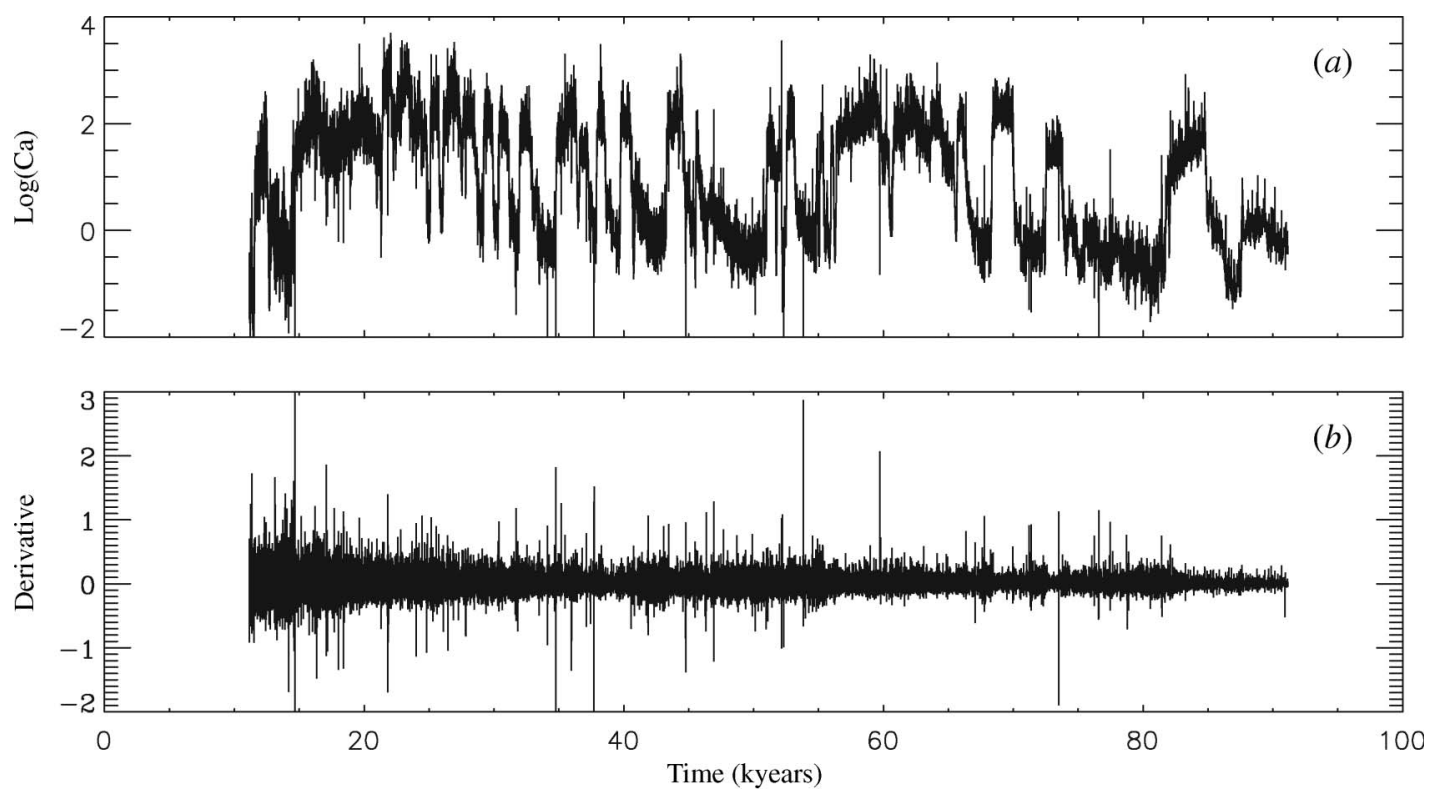

Figure 15. (a) The logarithm of the calcium concentration as a function of time (BP) in the GRIP ice core. The dating of this upper part of the record is rather precise. The temporal resolution is about 1 year, much better than the $\delta^{18} \mathrm{O}$ record since the dust does not diffuse in the ice. The signal is a proxy for the climatic state. (b) The derivative of the signal in $(a)$. This approximately stationary signal is strongly intermittent.

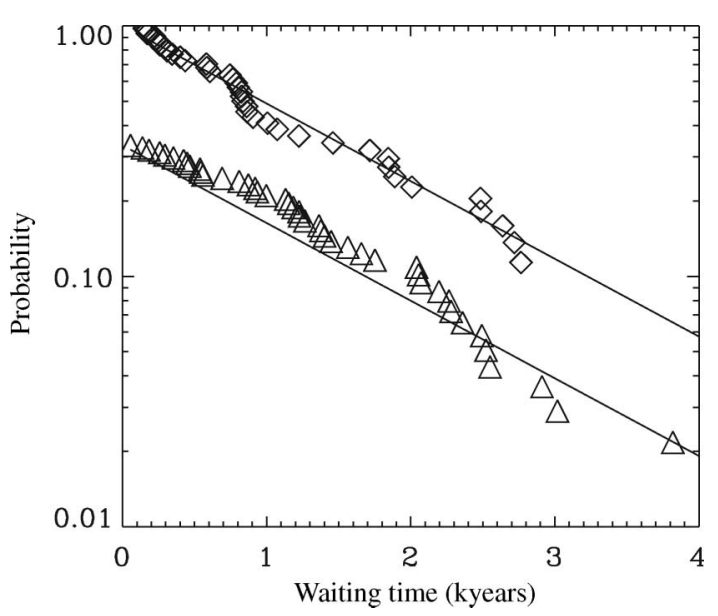

Figure 16. The jumping between the glacial and interstadial states is well described as a Poisson process. The waiting times are defined as the times between consecutive first up crossings through the level $\log (\mathrm{Ca})=2$ (glacial state) and first down crossings through the level $\log (\mathrm{Ca})=-0.6$ (interstadial state). The waiting times have an exponential distribution (diamonds). The full line is an exponential distribution with a mean waiting time of 1400 years. The data record is not long enough to determine if the waiting times for the interstadials and the glacial states are significantly different. The exponential distribution is consistent with the stochastic dynamics. The triangles, which are vertically shifted for clarity, are results from simulation (see text).

since the process (12) strictly speaking is not differentiable in time.

The stationary distribution for $x$ is a $t$-distribution which fits to the observed tail distribution for the noise

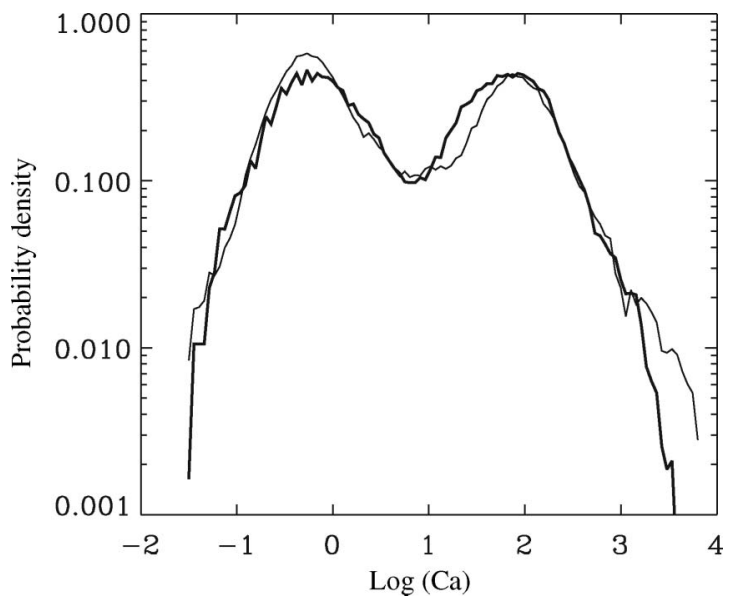

Figure 17. The probability density function (PDF) of the $\log (\mathrm{Ca})$ signal shows the bimodal distribution. The left maximum corresponds to the interstadial state and the right maximum corresponds to the glacial state. The thin curve is the PDF of the simulated signal (Figure 6).

on $y$. This term describes the forcing from the atmosphere. That the noise is white means that it is uncorrelated between consecutive data points. That does not exclude that the noise is red on shorter (unresolved) timescales. Actually the term $-x \mathrm{~d} t$ gives a correlation time of one year. It should be noted that the same signal could be scaled with a factor $\rho$ : $\mathrm{d} x=-\rho^{2} x \mathrm{~d} t+\rho\left(1+x^{2}\right)^{1 / 2} \mathrm{~d} B$ consistently with the data as long as $\rho>1 . \rho^{-1}$ signifies the correlation time (which is shorter than one year). The reason that 
this noise term is not just a Gaussian white noise term is that the intra-annual variability is strongly dependent on season, with much stronger intensity in winter than in summer, and that the inter-annual correlation leads to the red noise signal, where the 'nonGaussianity' survives the annual averaging.

The second noise term is an $\alpha$-stable noise with stability index $\alpha=1.75$ [45]. The $\alpha$-stable distributions, which are also called Levy flights, have cumulative probability tails which scale as $x^{-\alpha}$, for $\alpha<2$, implying that only moments of order less than $\alpha$ exists $\left(\left\langle|x|^{\beta}\right\rangle=\infty\right.$ for $\beta \geq \alpha$. The $\alpha$-stable distributions fulfill a generalised version of the central limit theorem, namely that the distributions of sums of identically distributed random variables with cumulative
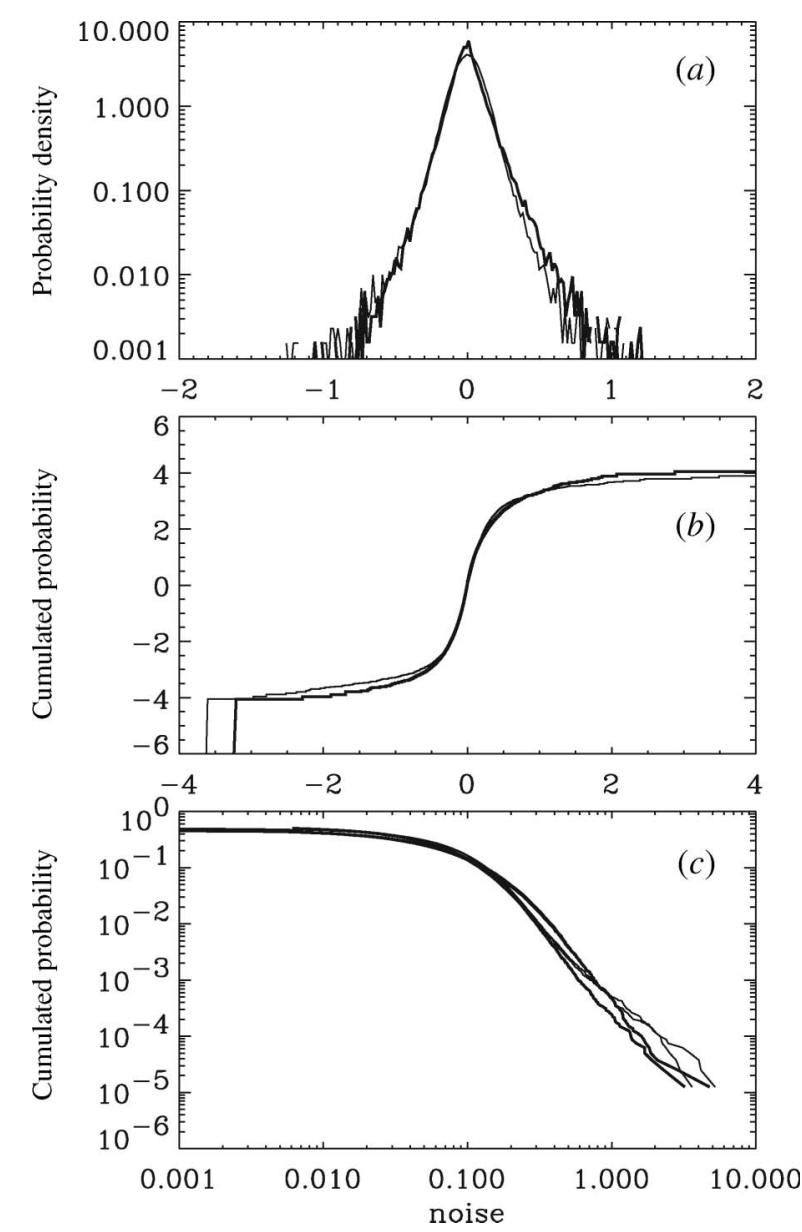

Figure 18. (a) The probability density of the noise (Figure $2(b))$. (b) The cumulated distribution of the noise. The scale is a 'probability paper scale' where a gaussian distribution shows up as a straight line. This signal is strongly nongaussian. (c) The two tails of (b) on a log-log plot. For the upper tail the probability of values larger than the abscissa is shown. The thin curves are from the simulation, showing that the signal is well described as containing a $t$-distributed noise component and an $\alpha$-stable noise component. distribution tails scaling as $x^{-\alpha 1 \rightarrow \alpha_{1}}$ converges to an $\alpha$-stable distribution with $\alpha=\alpha_{1}$. These distributions have very fat tails, meaning that the probability of extreme events is high, such that single extreme events within a period over which the variable is averaged will show up also in the distribution of the averages. The $\alpha-$ stable distributions were first observed in hydrological records of river flow [46], and have later been observed in various different physical systems [47] such as turbulent diffusion [48] and vortex dynamics [49]. To the present there is still no full theoretical understanding of why these distributions are observed, and to what extent it has importance in climate dynamics.

A generalisation of the Fokker-Planck equation for the two coupled Langevin equations with $\alpha$-stable noise excitations connects the stationary density solution to the pseudo-potential $U(y)$. However, only the marginal distributions are known. For $y$ this is the PDF for $\log (\mathrm{Ca})$ shown in Figure 17. The pseudopotential, shown in Figure 18, is thus determined iteratively by simulation starting from a solution to the stationary one-dimensional Fokker-Planck equation using the marginal distribution.

In order to validate that the $\log (\mathrm{Ca})$ signal can be described by (1) a consistency check must be performed. This is done by simulation. Using the derived pseudo-potential, Figure 19, fitting $\sigma_{1}$ and $\sigma_{2}$ from the noise structure of the signal, Figure 20, shows a realisation of (1). This should be compared with the $\log (\mathrm{Ca})$ signal, Figure $15(a)$.

The thin lines in Figures 17 and 18 are derived from the simulated signal. The stationary Fokker-Planck equation does not contain information about the timescales for jumping, Figure 16, and temporal harmonic decomposition of the sample as represented

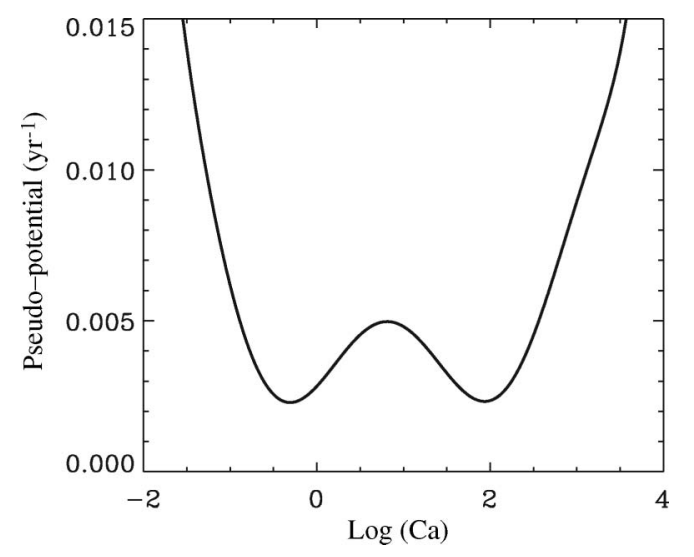

Figure 19. The climate pseudo-potential is a double-well potential with the left well representing the interstadial state and the right well representing the full glacial state. The potential is obtained from a generalised stationary FokkerPlanck equation. 


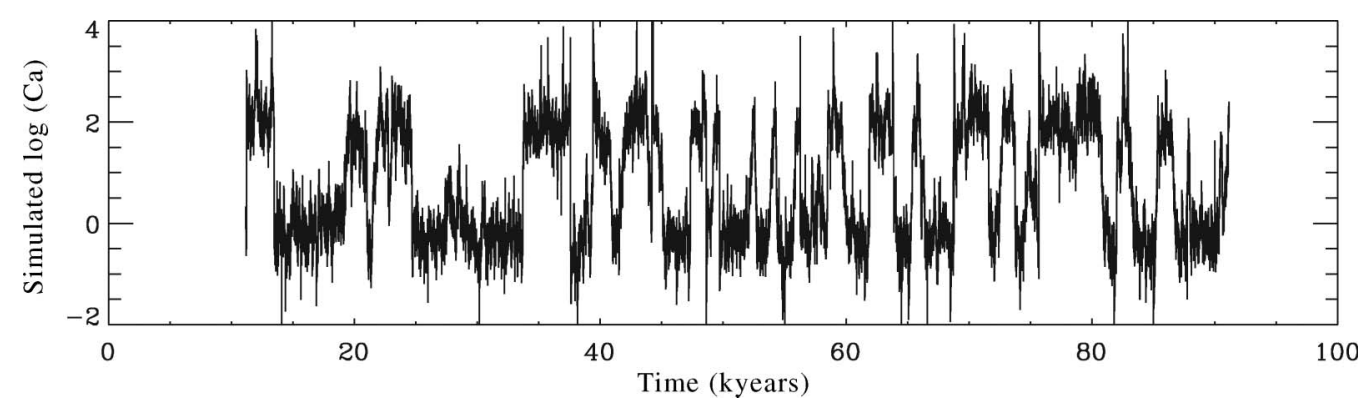

Figure 20. An artificial $\log (\mathrm{Ca})$ obtained from simulating a sample solution to the Langevin equation using the climate pseudopotential, an $\alpha=1.75$ white noise and $\sigma_{1} / \sigma_{2}=3$. This should be compared to Figure $16(a)$. The two signals are statistically similar, showing that the $\log (\mathrm{Ca})$ signal can be generated by the stochastic dynamics.

by the power spectra which are compared in Figure 21. These constitute independent verifications. As seen in all the figures the agreement is astonishing. Judged from different simulated realisations the two signals only deviate within the statistical uncertainty.

This is not merely an advanced curve fitting routine. If the calcium data is assumed to be generated by the dynamics described through a Langevin equation, the driving noise must be of the form described here. In order to understand the underlying climate dynamics it is important to establish the connection between this climatic proxy and the climate. It is especially important to interpret the two noise terms and connect them to the atmosphereocean dynamics. The noise term ' $\sigma_{1} \mathrm{~d} x$ ' is probably related to the 'normal' atmospheric fluctuations. Since the sampling is coarse on the timescales of these fluctuations, there is is an indeterminacy in the noise structure on timescales shorter than about one year. In the model this reflects itself in the invariance of this noise term with respect to $\rho$. The noise term $\sigma_{2} \mathrm{~d} L$ represents extreme events and calls for attention. The $\alpha$-stable noise seems to occur in dynamical systems with many different timescales where the dynamics becomes strongly intermittent.

\section{Stochastic resonance in climate transitions}

In the previous analysis the paleoclimatic record was described as resulting from noise induced jumping between two stable climate states, where the jumping probability only depends on the present state of the system. Thus, it is a Markov process, for which the distribution of waiting times becomes exponential, as indicated by the straight line in Figure 16. The observed record is short in the sense that only of the order $25 \mathrm{DO}$ events occurred in the last glacial period. The limited length of the record leaves the possibility that other models might fit the data just as well. It was recognised from spectral analysis that there seems to be a significant power at a frequency corresponding to a

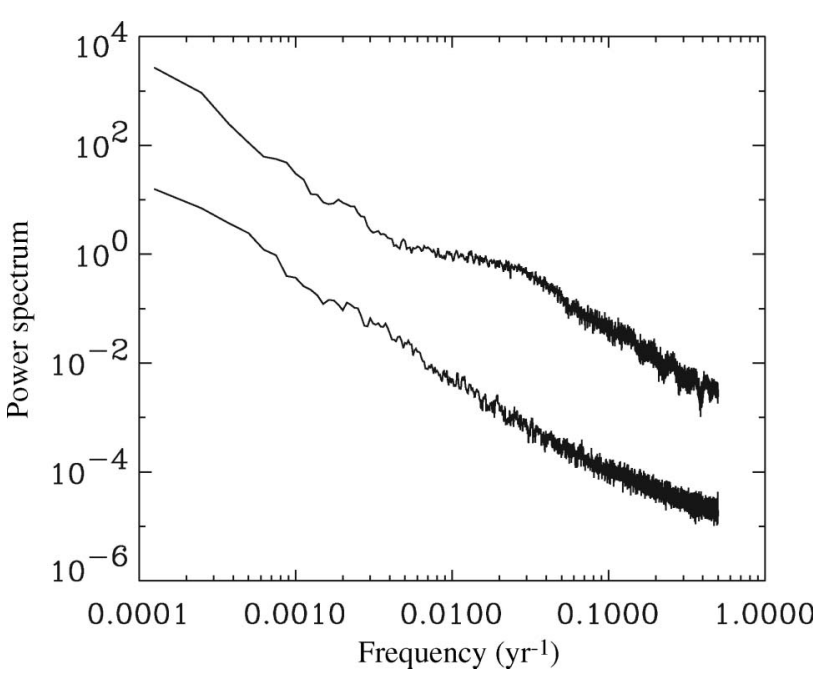

Figure 21. The temporal harmonic resolution as expressed through the power spectrum is an independent measure of the signal. The top curve is the power spectrum of $\log (\mathrm{Ca})$. This is a red noise spectrum without significant peaks. The bottom curve is the power spectrum of the simulated signal vertically shifted for clarity.

period of $1470 \mathrm{yr}$ for transitions into the interstadial climate state [50]. By fitting the phase and overlaying a periodic set of vertical lines on the climate record, see Figure 22, a striking regularity emerges [51]. This has been proposed to be due to a stochastic resonance response to an (unknown) periodic forcing [52] or a ghost resonance response to the beating frequency combination of two much shorter periods in the solar intensity [53].

The strikingly regular timing needs to be tested statistically. This is not completely straightforward: the general problem is that when observing a pattern in a data set, the significance of the pattern can be very difficult to assess a posteriori unless the space of possible outcomes for 'striking patterns' is known. 
We shall denote the identified time sequence for jumps as $t_{i}, i=1, \ldots, N$. A preferred periodicity in the time sequence can be detected by Rayleigh's $R$ measure defined as $R(\tau)=(1 / N)\left|\sum_{j} \exp 2 \pi t_{j} / \tau\right|$, where obviously $R(\tau) \in(0,1)$ [13]. This measure is easy to understand if we define the angles $\theta_{i}=2 \pi t_{i} / \tau$ and plot the angles on the unit circle. If the time sequence is multiples of the time $\tau$ modulo an (unknown) phase, all angles will be located near the same point on the unit circle and $R(\tau) \approx 1$. Conversely if the data points do not cluster on the unit circle we have $R(\tau) \approx 0$.

In Figure 23(b), the value of $R(\tau)$ as a function of $\tau$ is shown. The period of $1470 \mathrm{yr}$ shows the largest value $R=0.65$. The angles with respect to the $1470 \mathrm{yr}$ period of the time sequence of DO jumps are plotted on the unit circle in Figure 23(a). The mean phase is indicated by the radial line segments, the length is equal to $R$ $(1470 \mathrm{yr})$, indicated by the arrow in $(b)$, the mean phase defines the vertical lines plotted in Figure 22.
The next, and necessary, step in the analysis is to test the significance of the periodicity found in the data [54]. This can only be done by assuming a testmodel generating the data. Given such a model, we may choose any measure derived from the data, $x_{\mathrm{d}}$ to compare with the same measure derived from similar realisations of the test model, $x_{\mathrm{m}}$. The null hypothesis is then that the data series is a specific realisation of the model. It is important to note that a null hypothesis can only be rejected and not confirmed. That is, the value of the chosen measure for the data may well be within the high likelihood region for the model, but this does not prove that the data cannot be generated from another (competing) model with same high likelihood for the chosen measure. On the contrary, only if the measure for the data falls within a low likelihood region, say with probability measure $p \ll 1$, the model can be rejected with probability $1-p$.

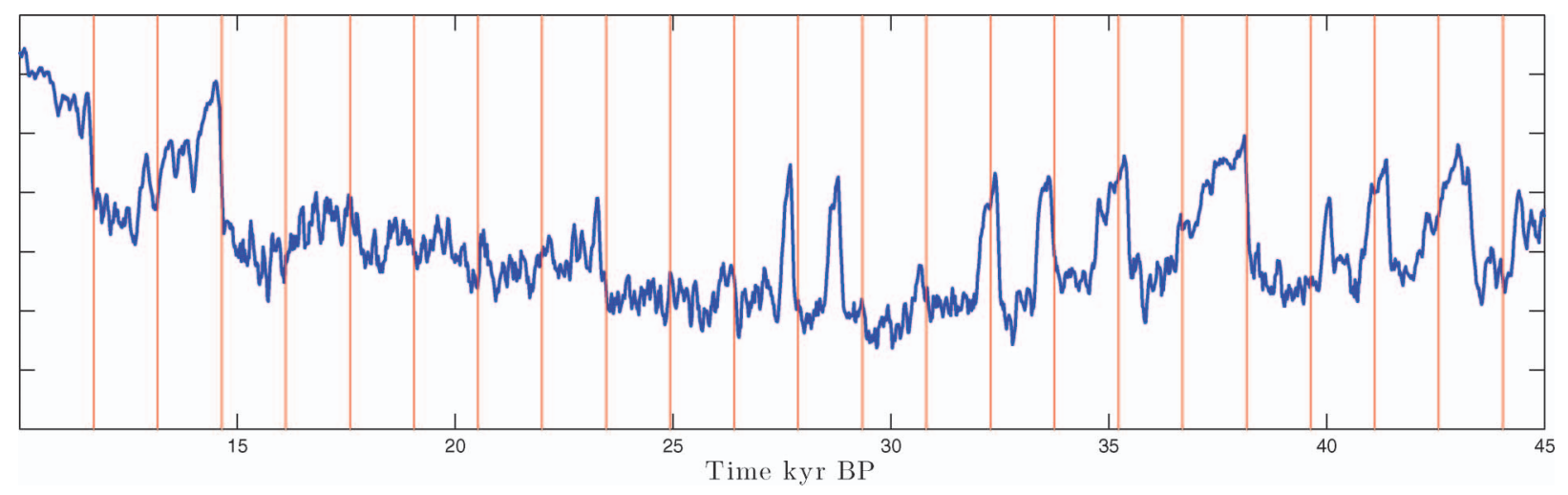

Figure 22. A part of the NGRIP oxygen-18 record, where the dating is done by annual layer counting from the top. This is a proxy for temperature, where the transition into the present warm period is seen to the left. The last 10 Dansgaard-Oeschger (DO) climate events are shown. The vertical red lines shows an apparent periodicity in the initiations of DO events. The significance of this regularity must be checked statistically.

(a)

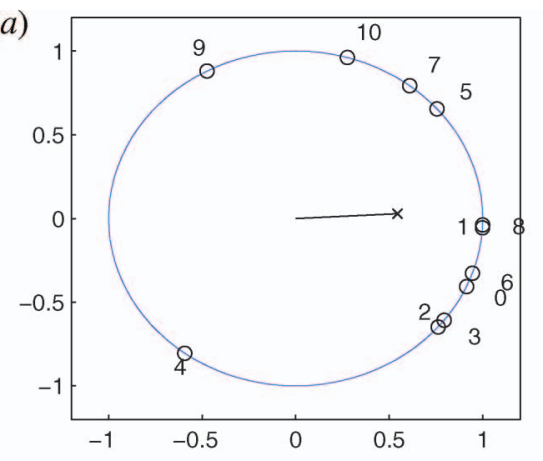

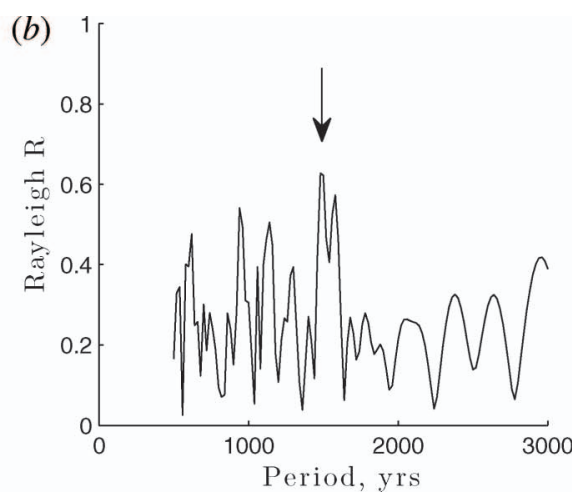

Figure 23. The Rayleigh $R$ for different periods. The maximum is obtained for the period $\tau=1470$ yr, indicated by the arrow. (a) Shows the timing of the onsets $t_{n}$ plotted on the unit circle using the transformation $\theta_{n}=2 \pi t_{n} / \tau$. The numbering is the standard numbering of DO events going back in time. The segments of radians points at the mean phase, corresponding to the vertical bars in Figure 22. 

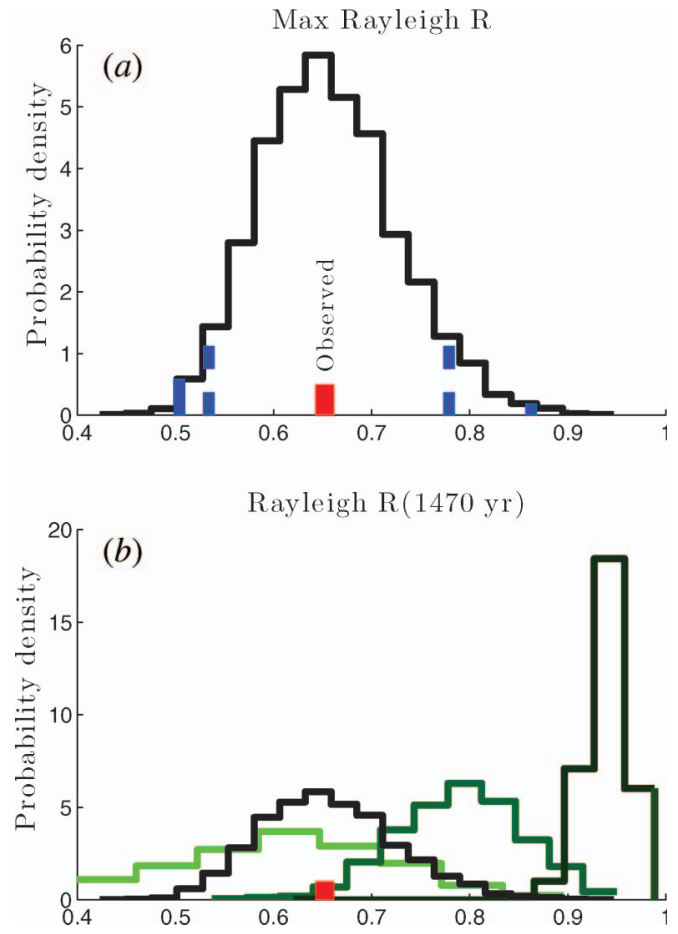

Figure 24. (a) By Monte Carlo an ensemble of 1000 realisations of waiting times in a $40 \mathrm{kyr}$ period has been generated from an exponential distribution with mean waiting time of 2800 years, corresponding to 14 DO-events in $40 \mathrm{kyr}$. This gives probability densities for the maximal Rayleigh's $R(\tau)$ in the range $500 \mathrm{yr}<\tau<5000 \mathrm{yr}$. The red bar gives the values for the observed ice-core record. The blue bars are $90 \%$ (dashed) and 99\% (full) confidence levels. (b) Same as in the top panel, with distribution functions obtained from stochastic resonance models with period of 1470 years. From light to dark green the model parameters are: $\mathrm{a}=0.1,0.2,0.4$ and $\sigma=0.38,0.35,0.27$ (see text), which generates on average $11 \mathrm{DO}$-events in $31 \mathrm{kyr}$. The important difference from the case shown in the panel above is that the Rayleigh's R in this case are calculated for the fixed period of $1470 \mathrm{yr}$. The red bar is the ice-core observation as above. The black curve is the distributions for the exponential model repeated from $(a)$. This shows that the SR model with $a=0.1$ cannot be identified in a sample of this length, since spurious coincidental periodicities will give a better match to the data than the $1470 \mathrm{yr}$ cycle.

The simplest possible model which can be chosen for the statistical test is that the DO events occur randomly, without a memory, on the millennial timescale. This is consistent with the analysis presented above. The waiting times follow an exponential distribution corresponding to a Poisson process. The mean waiting time can be assumed to be 2800 years. This is obtained as an estimate from the mean waiting times for the 14 DO events in the period 10-50 kyr. This is also the estimate obtained from the best fit to an exponential distribution of all DO events in the full glacial period shown as the straight lines in Figure 16.
To test the data against this model we use the Rayleigh R obtained from the data. For each of the measures a probability density for a sample, similar to the observed record, is obtained from a Monte Carlo generated ensemble of 10000 realisations. The result is shown together with the measure from the data record in Figure 24(a). From the figure it is obvious that the observations fall within the high likelihood region of the exponential distribution. The 90\% (dashed) and 99\% (full) confidence levels are shown in the figure as vertical blue bars. Thus there is no basis for rejecting the hypothesis of no-periodicity for the data.

The climate system is dominated by internal noise masking possible periodic components. Thus, a reasonable assumption is that a periodicity is caused by an internal non-linear amplification of a weak external periodic forcing. This could be described by a stochastic resonance as proposed by Alley et al. [52].

The stochastic resonance model [19] is defined by the governing equation:

$$
\begin{aligned}
\mathrm{d} x & =\partial_{x} U_{a}(x, t, \tau) \mathrm{d} t+\sigma \mathrm{d} B \\
& =\left\{-2\left(x^{3}-x\right)+a \cos (2 \pi t / \tau)\right\} \mathrm{d} t+\sigma \mathrm{d} B,
\end{aligned}
$$

where a particularly simple form of the drift potential $U_{a}(x, t, \tau)$ is chosen here. The potential is a double-well potential, which changes periodically with period $\tau$ from having a shallow well $(s)$ to the right and a deep well $(d)$ to the left to the opposite situation. The ratio of the barrier heights $H_{s \rightarrow d} / H_{d \rightarrow s}$ is determined by the amplitude parameter $a$. The timescales for jumping from the shallow well to the deep well is given by an Arrhenius formula; $T_{s \rightarrow d} \sim \exp \left(H_{s \rightarrow d} / 2 \sigma\right)$, and similarly for $T_{d \rightarrow s}$. The criterion for resonance, where the signal $x$ is most periodic, is $T_{s \rightarrow d} \ll \tau \ll T_{d \rightarrow s}$. This determines the noise intensity $\sigma$. The difference between a hysteresis loop through a set of bifurcations and a stochastic resonance is seen in comparing the schematics in Figures 25 and 26.

The proposition of rejecting a stochastic resonance (SR) model for the ice-core data is more tricky, since there exists a continuum of SR models with waiting time distributions from the exponential to the deltadistribution for the perfect periodicity [55]. However, the only spectral weight notably above the continuum is at $\tau^{-1}=1470 \mathrm{yr}^{-1}$ and not at the mean waiting time $2800 \mathrm{yr}^{-1}$. Near the stochastic resonance one should expect the same order of magnitude 'early jumps' (corresponding to a noise induced jump from the deep well to the shallow well) as 'late jumps' (corresponding to missing a jump from the shallow to the deep well). The mean waiting time being about 

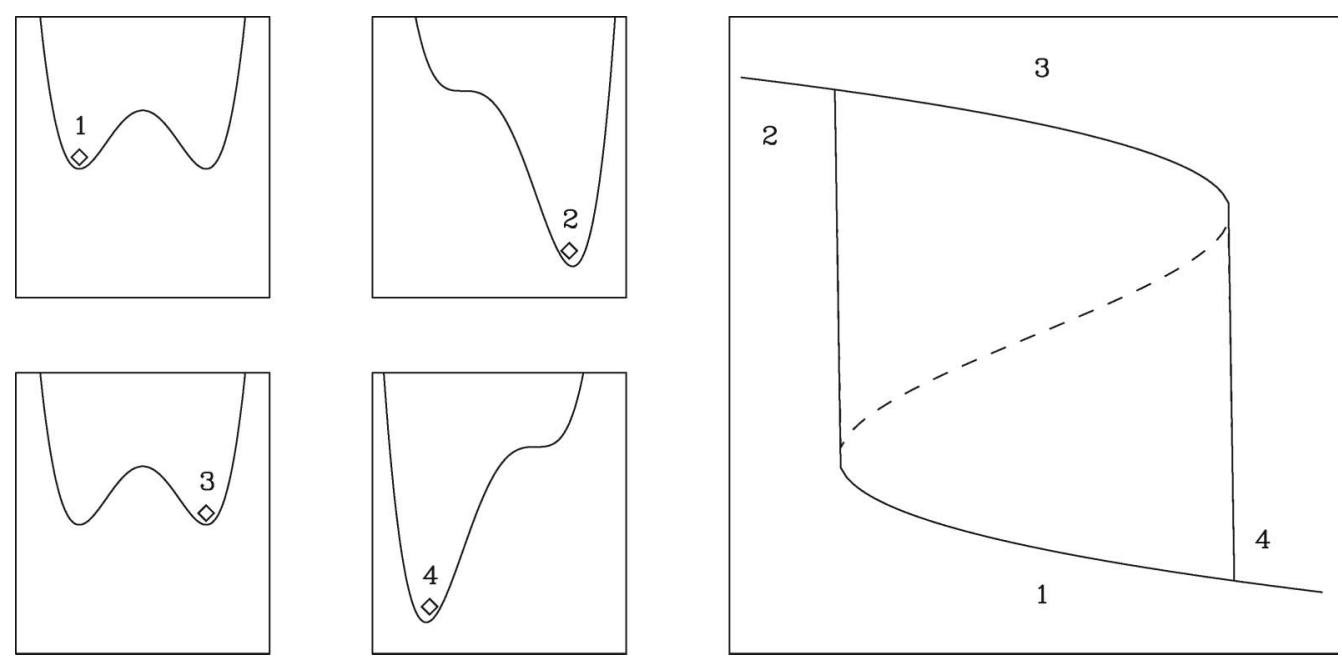

Figure 25. A strong periodic variation of the potential leads to hysteresis behaviour through successive saddle-note bifurcations.
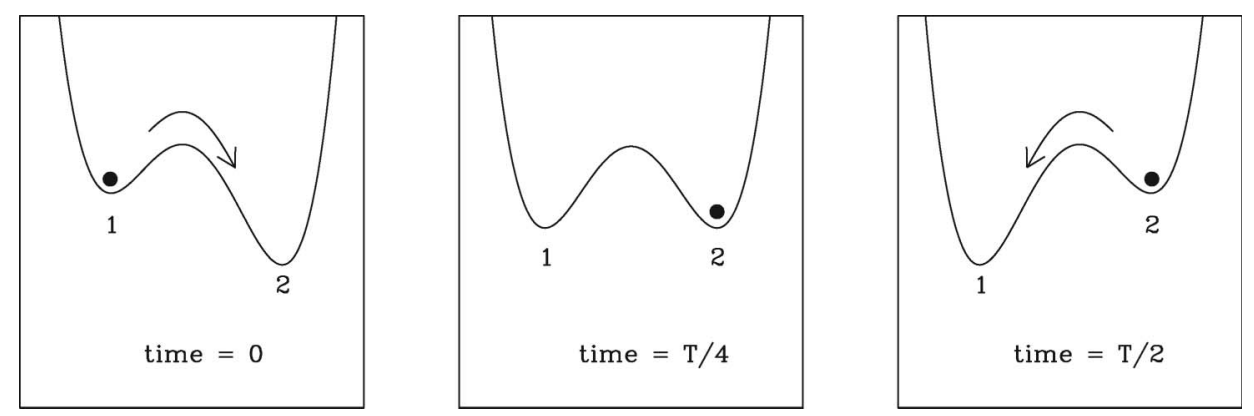

Figure 26. For a weak periodic variation of the potential internal noise is required to induce a transition. For the right combination of noise intensity and barrier height the response to the periodic change is still periodic. This is the stochastic resonance.

twice the observed spectral period indicates that a possible SR is 'off the resonance' with a too low noise level. In terms of SR parameters, this means that the criterion; $T_{s \rightarrow d} \ll \tau \ll T_{d \rightarrow s}\left(T_{s \rightarrow d}\right.$ being the mean waiting time for a transition from the shallow to the deep well), is not fulfilled. We rather see $\tau<T_{s \rightarrow d}$.

Here we test against three SR models with the period $\tau=1470 \mathrm{yr}$ and $a=0.1,0.2,0.4$. The mean number of DO events being 11 events/31 kyr corresponding to the climate record. This determines the noise intensity to be $\sigma=0.38,0.35,0.27$ for the three models.

An ensemble of 1000 simulations with same length as the data records were generated and the same three significance tests were performed. The results for the three models; $a=0.1$ (light green), $a=0.2$ (medium green), $a=0.4$ (dark green) are shown in Figure 24(b). The distribution in the top panel, for the exponential distribution, is over-plotted in black. It is seen that the first model, $a=0.1$, apparently has less periodicity, represented by Rayleigh's $R$, than the purely exponential model. This is because in the case of the SR model the distribution is of $R(1470 \mathrm{yr})$, while in the case of the exponential waiting time distribution (corresponding to $a=0$ ), the distribution is for the largest value of $R$ found in the sample. This means that for the SR model with $a=0.1$, the period will not be identified in comparison to other spurious coincidental periodicities. We have thus identified the 'weakest' SR model which may be identified for a sample of the size of the record.

So the statistical tests show that the waiting times for DO events are within the high likelihood region of the exponential distribution. This distribution implies that there is no long term memory in the climate system or unknown 1470 years periodic forcing triggering the climate shifts. By the nature of the statistical test we can only reject the hypothesis of a periodic component when the period is sufficiently above the noise level. For SR models with too low a strength of the periodic component, the period would with high probability not be detected in comparison to detecting a spurious coincidental periodicity in the sample. 


\section{Summary}

The paleoclimatic records show both remarkable climate stability on geological timescales and dramatic changes between different climate states. The transitions most likely results from combinations of destabilisation through bifurcations and transitions induced by internal variation, which can be well described as stochastic noise. The current state-of-the-art general circulation climate models do a fair job in integrating the flow equations for the atmosphere and oceans, admittedly at a coarse resolution. They also incorporate many physical and chemical interactions involving the cryosphere (ice masses), the lithosphere (the land masses) and the biosphere (vegetation) and give a realistic representation of the present climate. However, the models are far from being able to simulate the observed past climate transitions. It is even not known if the models possess a non-trivial bifurcation structure. The identification of the dynamical bifurcation diagram from observations should thus be a guideline for verification of realistic glacial climate models.

The presence of a fat tailed noise component could imply that the triggering mechanisms for climatic changes are rare extreme events. Such events, being on the timescale of seasons, are fundamentally unpredictable and never captured in the numerical circulation models. The lack of dynamical range might be due to underestimation of internal variability in too coarse resolution, thus the climate noise is too weak to induce transitions from one stable climate state to another. This could be part of the explanation why these models have yet never succeeded in simulating shifts between climatic states.

\section{Notes on contributor}

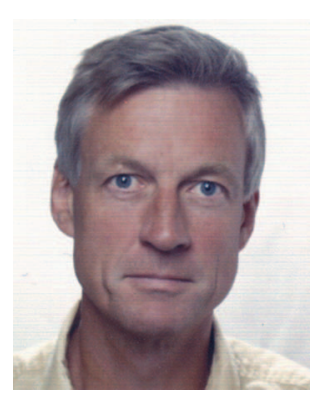

Peter Ditlevsen is Professor of Theoretical Physics at the Niels Bohr Institute, University of Copenhagen. His main interests are in statistical physics, turbulence and climate dynamics. His involvement in climate research began when he was researcher for a year at the Danish Meteorological Institute. Since then he has become deeply involved in interpretation of ice-core records obtained from drilling in Greenland. He obtained his Ph.D. from Denmark's Technical University in solid state physics and did his Doctorate at the University of Copenhagen in turbulence and climate dynamics.

\section{References}

[1] M.I. Bodyko, The effect of solar ratiation variations on the climate of the Earth, Tellus 21 (1969), pp. 611-619.

[2] W.D. Sellers, A global climatic model based on the energy balance of the Earth-atmosphere system, J. Appl. Meteorol. 8 (1969), pp. 392-400.
[3] P.F. Hoffman, A.J. Kaufman, G.P. Halverson, and D.P. Schrag, A neoproterozoic Snowball Earth, Science 281 (1998), pp. 1342-1346.

[4] J.C.G. Walker, P.B. Hays, and J.F. Kasting, A negative feedback mechanism for the long-term stabilization of Earth's surface temperature, J. Geophys. Res. 86 (1981), pp. 9776-9782.

[5] P.D. Ditlevsen, A climatic thermostat making Earth habitable, Int. J. Astrobiol. 4 (2005), pp. 3-7.

[6] K. Hasselmann, Stochastic climate models, Tellus 28 (1976), pp. 473-485.

[7] C.W. Gardiner, Handbook of Stochastic Methods, Springer Verlag, New York, 1985.

[8] W. Dansgaard, S.J. Johnsen, H.B. Clausen, D. DahlJensen, N.S. Gundestrup, C.U. Hammer, C.S. Hvidberg, J.P. Steffensen, A.E. Sveinbjornsdottir, J. Jouzel, and G. Bond, Evidence for general instability of past climate from a 250-kyr ice-core record, Nature 364 (1993), pp. 218-220.

[9] P. Huybers, Glacial variability over the last 2Ma: an extended depth-derived age model, continuous obliquity pacing, and the Pleistocene progression, Quat. Sci. Rev. 26 (2007), pp. 37-55.

[10] J. Hays, J. Imbrie, and N. Shackleton, Variations in Earths's orbit: pacemaker of the ice ages, Science 194 (1976), pp. 1121-1132.

[11] M. Milankovitch, Mathematiche Klimalehre und Astronomiche Theorie der Klimaschwankungen, Handbuch der Klimalogie Band 1 Teil A, Borntrager, Berlin, 1930.

[12] J. Imbrie, A. Berger, E.A. Boyle, S.C. Clemens, A. Duffy, W.R. Howard, G. Kukla, J. Kutzbach, D.G. Martinson, A. McIntyre, A.C. Mix, B. Molfino, J.J. Morley, L.C. Peterson, N.G. Pisias, W.L. Prell, M.E. Raymo, N.J. Shackleton, and J.R. Toggweiler, On the structure and origin of major glacial cycles. 2. The 100,000-year cycle, Paleoceanography 8 (1993), pp. 699-735.

[13] P. Huybers and C. Wunsch, Obliquity pacing of the late Pleistocene glacial terminations, Nature 434 (2005), pp. 491-494.

[14] M.E. Raymo, W.F. Ruddiman, J. Backman, B.M. Clement, and D.G. Martinson, Late Pleiocene variations in Northern hemisphere ice sheets and North Atlantic deep water circulation, Paleoceanography 4 (1989), pp. 413-446.

[15] H. Gildor and E. Tziperman, Sea ice as the glacial cycles' climate switch: role of seasonal and orbital forcing, Paleoceanography 15 (2000), pp. 605-615.

[16] E. Källen, C. Crafoord, and M. Ghil, Free oscillations in a climate model with ice-sheet dynamics, J. Atmos. Sci. 36 (1979), pp. 2292-2303.

[17] B. Saltzman and A. Sutera, The mid-quaternary climate transition as the free response of a three-variable dynamical model, J. Atmos. Sci. 44 (1987), pp. 236-241.

[18] H. LeTreut and M. Ghil, Orbital forcing, climate interactions, and glacial cycles, J. Geophys. Res. 88 (1983), pp. 5167-5190.

[19] R. Benzi, G. Parisi, A. Sutera, and A. Vulpiani, Stochasic resonance in climate change, Tellus 34 (1982), pp. 10-16.

[20] J. Imbrie, E.A. Boyle, S.C. Clemens, A. Duffy, W.R. Howard, G. Kukla, J. Kutzbach, D.G. Martinson, A. McIntyre, A.C. Mix, B. Molfino, J.J. Morley, L.C. Peterson, N.G. Pisias, W.L. Prell, M.E. Raymo, N.J. Shackleton, and J.R. Toggweiler, On the structure and origin of major glacial cycles. 1. Linear responses to Milankovitch forcing, Paleoceanlography 7 (1992), pp. 701-738. 
[21] M.A. Kominz and N.G. Pisias, Pleistocene climate: deterministic or stochastic, Science 204 (1979), pp. 171-172.

[22] Y. Ashkenazy and E. Tziperman, Are the $41 \mathrm{kyr}$ oscillations a linear response to Milankovitch forcing? Quat. Sci. Rev. 23 (2004), pp. 1879-1890.

[23] B. Saltzman and K.A. Maasch, A first-order global model of late Cenozoic climatic change, Climate Dyn. 5 (1991), pp. 201-210.

[24] P.U. Clark and D. Pollard, Origin of the middle Pleistocene transition by ice sheet erosion of regolith, Paleoceanography 13 (1998), pp. 1-9.

[25] P. Huybers and E. Tziperman, Integrated summer insolation forcing and 40,000 year glacial cycles: the perspective from an icesheet/energy-balance model, $\mathrm{Pa}$ leoceanography 23 (2008). PAI208.

[26] D. Paillard, The timing of Pleistocene glaciations from a simple multiple-state climate model, Nature 391 (1998), pp. 378-381.

[27] P.D. Ditlevsen, The bifurcation structure and noise assisted transitions in the Pleistocene glacial cycles, to appear in Paleoceanography.

[28] P. Huybers and I. Eisenman, Integrated summer insolation calculations. NOAA/NCDC Paleoclimatology Program, Data Contribution \# 2006-079, 2006. Available at http://www.ncdc.noaa.gov/paleo/forcing.html

[29] S.W. Broecker. Terminations Milankovitch and Climate: Understanding the Response to Astronomical Forcing, A. Berger, J. Imbrie, J. Hays, G. Kukla, and B. Saltzman, eds., Reidel Publishing Company, Dordrecht-Holland, 1984, pp. 687-698.

[30] M.E. Raymo, The timing of major climate terminations, Paleoceanography 12 (1997), pp. 577-585.

[31] P.D. Ditlevsen, Observation of alpha-stable noise and a bistable climate potential in an ice-core record, Geophys. Res. Lett. 26 (1999), pp. 1441-1444.

[32] G. Bond, W. Broecker, S. Johnsen, J. McMagnus, L. Labeyrie, J. Jouzel, and G. Bonani, Correlations between climate records from North Atlantic sediments and Greenland ice, Nature 365 (1993), pp. 143-147.

[33] J.W. Beck, J. Recy, F. Taylor, R.L. Edwards, and G. Cabioch, Abrupt changes in early Holocene tropical sea surface temperature derived from coral records, Nature 385 (1997), pp. 705-707.

[34] W.S. Broecker, D.M. Peteet, and D. Rind, Does the ocean-atmosphere system have more than one stable mode of operation? Nature 315 (1985), pp. 21-26.

[35] P. Cessi, A simple box model of stochastically forced thermohaline flow, J. Phys. Oceanogr. 24 (1994), pp. 1911-1920.

[36] S. Rahmstorf, Bifurcations of the Atlantic thermohaline circulation in response to changes in the hydrological cycle, Nature 378 (1995), pp. 145-149.

[37] J. Pedlosky, Ocean Circulation Theory, Springer-Verlag, Berlin, 1998.

[38] P. Welander, Thermohaline effects in the ocean circulation and related simple models, in Large-Scale Transport Processes in Oceans and Atmosphere, J. Willebrand and D.L.T. Anderson, eds., Publishing Company, Dordrecht, 1986, pp. 163-200.

[39] H. Stommel, Thermohaline convection with two stable regimes of flow, Tellus 13 (1961), pp. 224-230.
[40] J.C. Duplessy, N.J. Shackleton, R.G. Fairbanks, L. Labeyrie, D. Oppo, and N. Kallel, Deepwater source variations during the last climate cycle and their impact on the global deepwater circulation, Paleoceanography 3 (1988), pp. 343-360.

[41] T.F. Stocker and D.G. Wright, Rapid changes in ocean circulation and atmospheric radiocarbon, Paleoceanography 11 (1996), pp. 773-795.

[42] W.S. Broecker, Thermohaline circulation, the Achilles heel of our climate system: will man-made $\mathrm{CO}_{2}$ upset the current balance?, Science 278 (1997), pp. 15821588.

[43] K. Fuhrer, A. Neftel, M. Anklin, and V. Maggi, Continuous measurements of hydrogen peroxide, formaldehyde, calcium and ammonium concentrations along the new GRIP ice core from Summit, Central Greenland, Atmos. Environ. Part A 27 (1993), pp. 1873-1880.

[44] P. Yiou, K. Fuhrer, L.D. Meeker, J. Jouzel, S. Johnsen, and P.A. Mayewski, Paleoclimatic variability inferred from the spectral analysis of Greenland and Antarctic icecore data, J. Geophys. Res. 102 (1997), pp. 26441-26454.

[45] G. Samorodnitsky and M.S. Taqqu, Stable Nongaussian Random Processes, Chapman and Hall, New York, 1994.

[46] H.E. Hurst, Long term storage capacity of reservoirs, Trans. Am. Soc. Civil Eng. 116 (1951), pp. 770-808.

[47] M.F. Shlesinger, M.G. Zaslavsky, and U. Frisch, Levy Flights and Related Topics in Physics, Springer Verlag, Berlin, 1994.

[48] G. Zimbardo, P. Veltri, G. Basile, and S. Pricipato, Anomalous diffusion and Levy random-walk of magneticfield lines in 3-dimensional turbulence, Phys. Plasmas 2 (1995), pp. 2653-2663.

[49] J.A. Viecelli, Statistical-mechanics and correlation-properties of a rotating 2-dimensional flow of like-sign vortices, Phys. Fluids A 5 (1993), pp. 2484-2501.

[50] P.M. Grootes and M. Stuiver, Oxygen 18/16 variability in Greenland snow and ice with $10^{-3}$ - to $10^{5}$-year time resolution, J. Geophys. Res. 102 (1997), pp. 2645526470.

[51] M. Schulz, The tempo of climate change during Dansgaard-Oeschger interstadials and its potential to affect the manifestation of the 1470-year climate cycle, Geophys. Res. Lett. 29 (2002). 1029/2001GL 013277.

[52] R.B. Alley, S. Anandakrishnan, and P. Jung, Stochastic resonance in the North Atlantic, Paleoceanography 16 (2001), pp. 190-198.

[53] H. Braun, M. Christi, S. Rahmstorf, A. Ganopolski, A. Mangini, C. Kubatski, K. Roth, and B. Kromer, Possible solar origin of the 1,470-year glacial climate cycle demonstrated in a coupled model, Nature 438 (2005), pp. 208-211.

[54] P.D. Ditlevsen, K.K. Andersen, and A. Svensson, The DO-climate events are probably noise induced: statistical investigation of the claimed 1470 years cycle, Climate of the Past 3 (2007), pp. 129-134.

[55] P.D. Ditlevsen, M.S. Kristensen, and K.K. Andersen, The recurrence time of Dansgaard-Oeschger events and limits on the possible periodic component, J. Climate 18 (2005), pp. 2594-2603. 